\title{
Handling Qualities of Model Reference Adaptive Controllers with Varying Complexity for Pitch-Roll Coupled Failures
}

\author{
Jacob Schaefer ${ }^{1}$, Curt Hanson ${ }^{2}$ \\ NASA Dryden Flight Research Center, Edwards, CA, United States, 93523 \\ Marcus A. Johnson ${ }^{3}$ \\ TYBRIN Corporation, Edwards, CA, United States, 93523 \\ Nhan Nguyen ${ }^{4}$ \\ NASA Ames Research Center, Mountain View, CA, United States, 94035
}

\begin{abstract}
Three model reference adaptive controllers (MRAC) with varying levels of complexity were evaluated on a high performance jet aircraft and compared along with a baseline nonlinear dynamic inversion controller. The handling qualities and performance of the controllers were examined during failure conditions that induce coupling between the pitch and roll axes. Results from flight tests showed with a roll to pitch input coupling failure, the handling qualities went from Level 2 with the baseline controller to Level 1 with the most complex MRAC tested. A failure scenario with the left stabilator frozen also showed improvement with the MRAC. Improvement in performance and handling qualities was generally seen as complexity was incrementally added; however, added complexity usually corresponds to increased verification and validation effort required for certification. The tradeoff between complexity and performance is thus important to a controls system designer when implementing an adaptive controller on an aircraft. This paper investigates this relation through flight testing of several controllers of vary complexity.
\end{abstract}

\section{Nomenclature}

A $=$ matrix of state derivative coefficients

$\mathrm{B}=$ matrix of control effectiveness derivatives

$\mathrm{CH}=$ Cooper-Harper

FAA $=$ Federal Aviation Administration

FAST $=$ Full-scale Advanced Systems Testbed

HUD $=$ heads-up display

$I \quad=$ inertia matrix

ILS $=$ instrument landing system

IRAC $=$ Integrated Resilient Aircraft Control

MRAC $=$ model reference adaptive controller

NASA $=$ National Aeronautics and Space Administration

NDI $=$ nonlinear dynamic inversion

$n=$ order of reference model

$\mathrm{OCM}=$ optimal control modification

PIO $=$ pilot induced oscillation

$p \quad=$ roll rate $(\mathrm{deg} / \mathrm{s})$

$q \quad=$ pitch rate $(\mathrm{deg} / \mathrm{s})$

$R \quad=$ gain for normalization of learning rate

\footnotetext{
${ }^{1}$ Aerospace Engineer, Flight Controls Branch, P.O. Box 273/Mail Stop 4840D, AIAA Member.

${ }^{2}$ Aerospace Engineer, Flight Controls Branch, P.O. Box 273/Mail Stop 4840D, AIAA Member.

${ }^{3}$ Aerospace Engineer, Flight Controls Branch, P.O. Box 273/Mail Stop 4840D, AIAA Member.

${ }^{4}$ Research Scientist, Intelligent Systems Division, Mail Stop 269-1, AIAA Associate Fellow.
} 


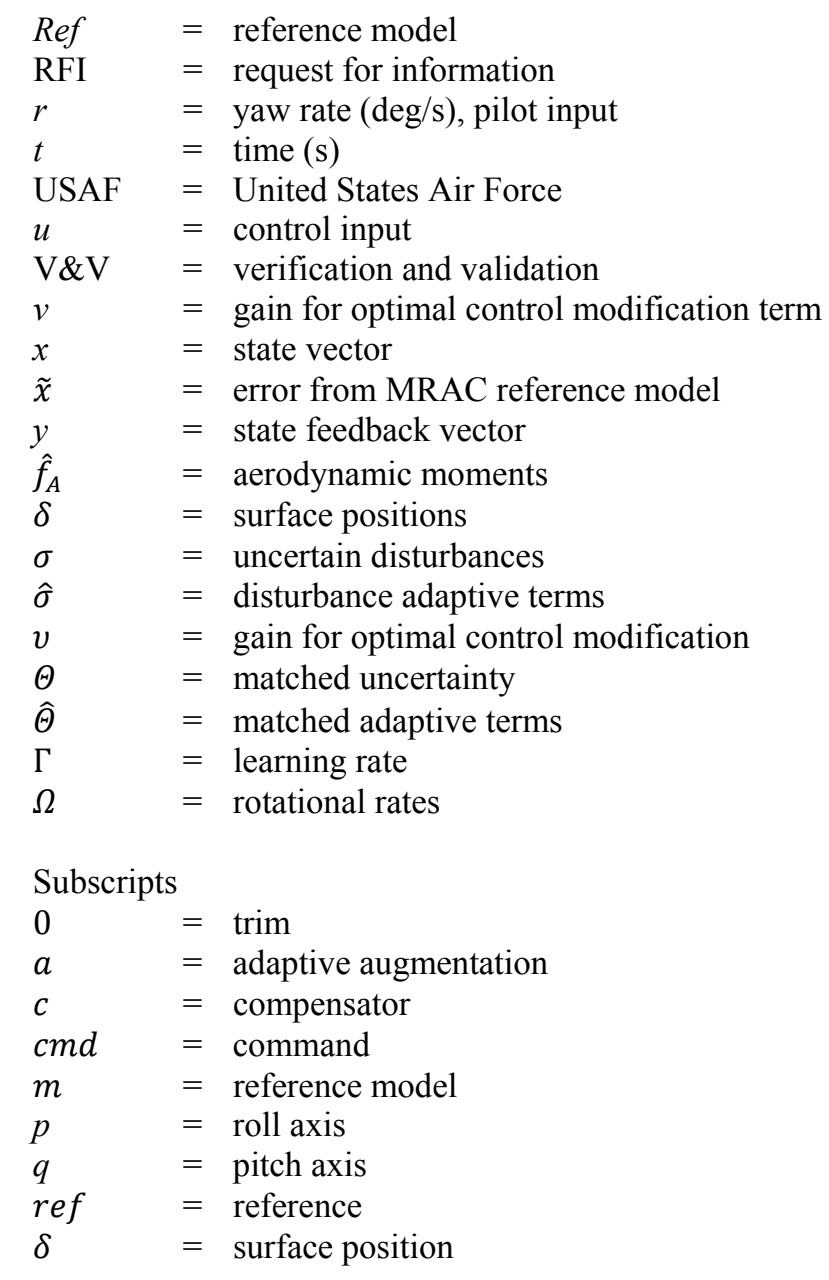

\section{Introduction}

Glight tests of several versions of a model-reference adaptive controller (MRAC) were carried out on the National C Aeronautics and Space Administration's (NASA) Full-scale Advanced Systems Testbed (FAST), which is an F/A-18 (McDonnell Douglas, now The Boeing Company, Chicago, Illinois) high performance jet aircraft shown in Fig. 1. This aircraft has a research flight control system that allows for testing of experimental control laws. Further details on the FAST aircraft can be found in (Ref. 1).

In 2009 a request for information (RFI) was released by NASA's Integrated Resilient Aircraft Control (IRAC) project seeking ideas for adaptive control experiments for loss of control events. ${ }^{2}$ One of the focus areas identified from the responses NASA received was to investigate simple yet effective adaptive control algorithms to help address the issue of verification and validation $(\mathrm{V} \& \mathrm{~V})$ testing of adaptive flight controls. Adaptive controllers, such as the MRAC, face many difficulties with regards to being certified and implemented on civilian or military aircraft. There are large gaps in the certification process for dealing with adaptive controllers. ${ }^{3}$ To date; the Federal Aviation Administration (FAA) has not certified any adaptive controllers for commercial aircraft. ${ }^{3}$ Part of the process to certification is $\mathrm{V} \& \mathrm{~V}$ testing. Adaptive controllers can become complex with many adaptive terms and complex update laws. The required testing to prove that the controller will behave appropriately under all situations, while also providing beneficial results in the presence of failures, can require extensive testing. By simplifying the controllers, the required testing could potentially be greatly reduced. Simplification is often at the expense of performance and the controller's ability to handle failure conditions. So there exists a tradeoff between the complexity and performance of a controller. 


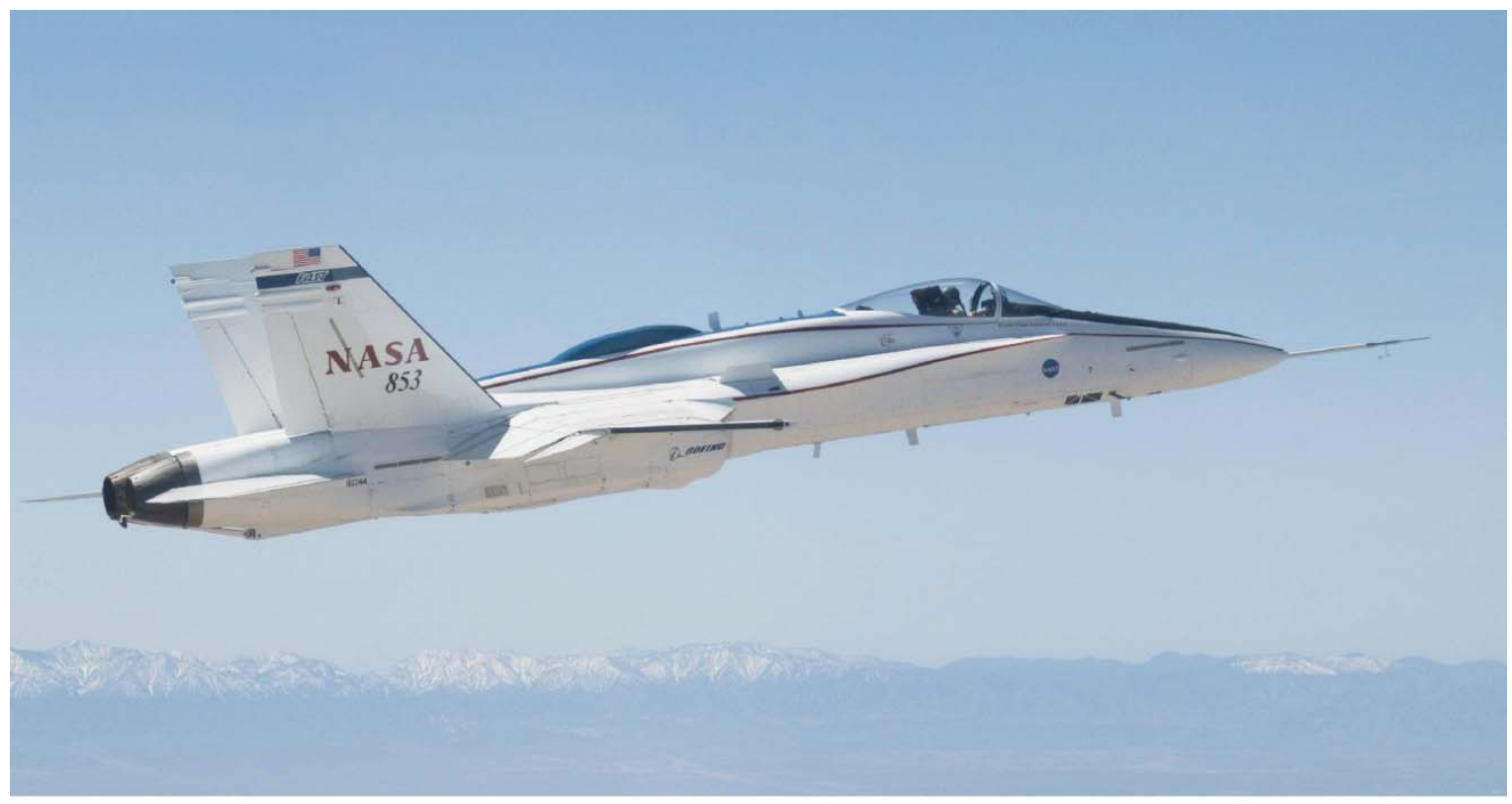

Figure 1. FAST aircraft.

There are many different techniques in literature for adaptive control with varying degrees of complexity, but there is little guidance for the flight control designer regarding the appropriate level of complexity to choose. This paper presents results from flight tests by starting with a baseline nonlinear dynamic inversion controller, then adding on to it a very basic model reference adaptive controller, and then adding in complexity elements. Using performance metrics and handling qualities ratings for specific tasks, these three adaptive controllers were evaluated and compared to the baseline nonlinear dynamics inversion control law. These controllers were evaluated under failure conditions that include a roll to pitch input coupling condition and a frozen left stabilator failure.

In this paper, the controller designs are presented. The failure conditions that the controllers were tested to are outlined. The performance and evaluation methods used for analysis of the controllers are described. Flight test results are given, along with a discussion regarding the tradeoff between controller complexity and performance.

\section{Description of Controllers}

Three model reference adaptive controllers were designed to be added onto a baseline nonlinear dynamic inversion controller (NDI). The MRACs had varying complexity by changing the number of adaptive terms and by changing the update laws.

\section{A. Baseline Controller (NDI)}

A nonlinear dynamic inversion controller is used as a baseline controller to provide stability and good aircraft handling qualities for the healthy, unfailed FAST aircraft. ${ }^{4}$ Figure 2 shows the NDI controller integrated with the MRAC adaptive augmentation. 


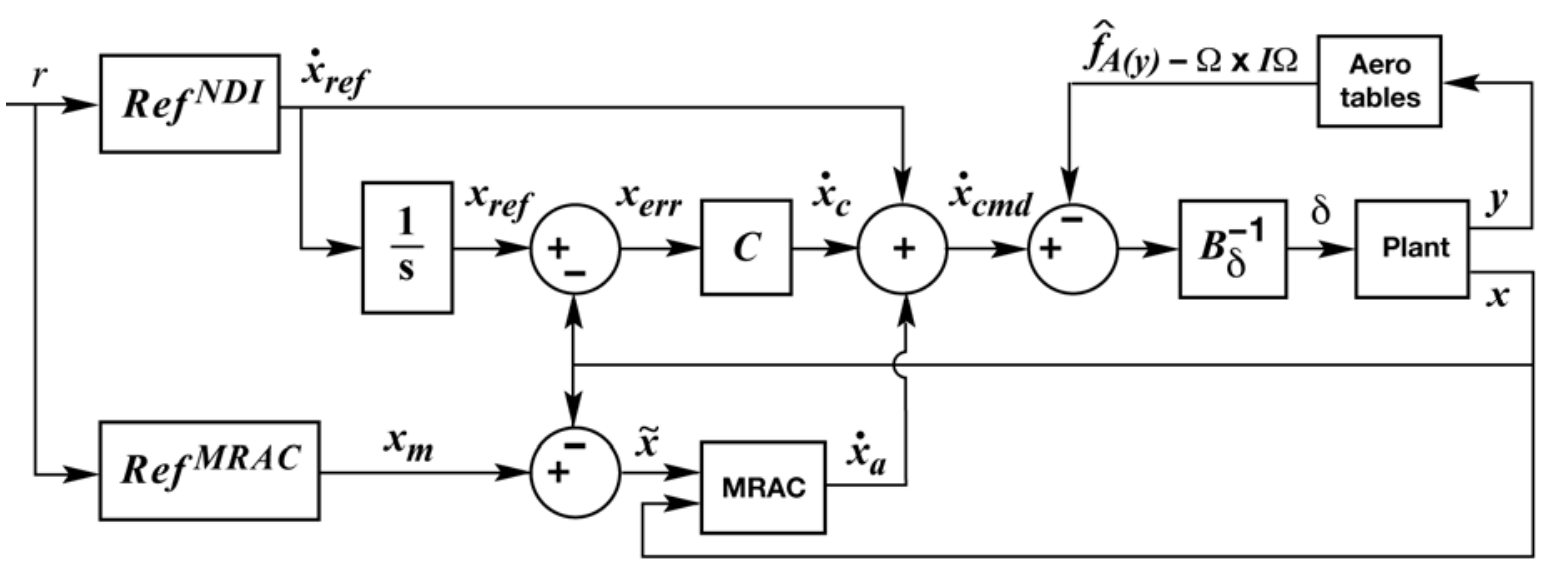

Figure 2. NDI and MRAC block diagram.

A total of ten control surface commands necessary to achieve the desired dynamics are calculated using Eq. (1). ${ }^{4}$ The vector of estimated aerodynamic moments $\hat{f}_{A}(y(t))$ is calculated from on-board aerodynamic lookup tables while rotational rates $\Omega(t)$ are measured using aircraft sensors. $I$ is the inertia matrix of the aircraft. Predetermined trim surface commands appropriate for the test flight condition are specified by $\delta_{0}$ and a weighted pseudo-inverse is used to compute $B_{\delta}^{-1}$, the inverse of the control effectiveness derivatives with respect to the surface positions weighted by a control allocation matrix.

$$
\delta(t)=B_{\delta}^{-1}\left(I \dot{x}_{c m d}(t)+\Omega(t) \times I \Omega(t)-\hat{f}_{A}(y(t))\right)+\delta_{0}
$$

The rotation acceleration commands $\dot{x}_{c m d}(t)$ contain the sum of the desired reference dynamics $\dot{x}_{r e f}(t)$, the output of an error compensator $\dot{x}_{c}(t)$, and adaptive control augmentation $\dot{x}_{a}(t)$ as described by Eq. (2). Note that the adaptive controller only augments the pitch and roll axes.

$$
\dot{x}_{c m d}(t)=\dot{x}_{r e f}(t)+\dot{x}_{c}(t)+\dot{x}_{a}(t)=\left[\begin{array}{l}
\dot{p}_{r e f}(t)+\dot{p}_{c}(t)+\dot{p}_{a}(t) \\
\dot{q}_{r e f}(t)+\dot{q}_{c}(t)+\dot{q}_{a}(t) \\
\dot{r}_{r e f}(t)+\dot{r}_{c}(t)
\end{array}\right]
$$

There are separate reference models for the NDI and MRAC controllers, each driven by the pilot's control input $r(t)$. The NDI reference models, $R e f^{N D I}$, generally represent the same desired dynamics as the MRAC reference models, $R e f^{M R A C}$, but may be modified to simulate a failed or damaged aircraft. The state feedback vector $y(t)$ contains measurements of the inputs required for the aerodynamic lookup tables, such as angle of attack and velocity, as well as the rotational rates.

\section{B. Model Reference Adaptive Controller}

Model reference adaptive control derives its name from the use of reference model dynamics to define a desired trajectory for the system outputs to follow. ${ }^{5}$ When the outputs do not follow the desired dynamics, the MRAC augments the NDI by adding dynamics in an attempt to obtain the desired dynamics of the reference model. The reference model for the aircraft axis of interest is specified as a stable, linear time-invariant system as in Eq. (3). The pitch axis reference model is second order, representing the desired short-period dynamics. The roll axis reference model is first order, and no adaptive augmentation is applied in the yaw axis.

$$
\dot{x}_{m}(t)=A_{m} x_{m}(t)+B_{m} r(t)
$$


Constant matrices $A_{m} \in \mathbb{R}^{n \times n}$ and $\mathrm{B}_{m} \in \mathbb{R}^{n \times 1}$ are selected to give good aircraft handling qualities. The aircraft's true, unknown dynamics are written as shown in Eq. (4),

$$
\dot{x}(t)=A x(t)+B_{m}(u(t)-\sigma(t))
$$

where $\sigma(t)$ is a scalar, time-varying uncertain disturbance. The unknown aircraft dynamics and known reference model dynamics are related according to the matching condition shown in Eq. (5),

$$
A=A_{m}-B_{m} \Theta^{T}
$$

The order $n$ of the reference model defines the number of matched uncertainties in the parameter vector $\Theta$. These additive uncertainties are commonly referred to as A-matrix uncertainties and are assumed to be either constant or slowly varying in comparison to the aircraft's dynamics. The time-varying disturbance term $\sigma(t)$ can be used to model coupled dynamics between axes. A scalar control input $u(t)$, given in Eq. (6), is computed as a combination of the reference input and augmentation from the adaptive controller. Note that, $r(t)$, the reference command component of the control signal, is provided by the NDI controller. ${ }^{6}$

$$
u(t)=r(t)+\dot{x}_{a}(t)=r(t)+\widehat{\Theta}^{\mathrm{T}}(t) x(t)+\hat{\sigma}(t)
$$

$\widehat{\Theta}(t)$ and $\widehat{\sigma}(t)$ are adaptive parameters to be estimated. Eq. (7) is formulated by taking the control input from Eq. (6) and substituting it into the true aircraft dynamics of Eq. (4) along with the matching condition of Eq. (5).

$$
\dot{x}(t)=A_{m} x(t)+B_{m} r(t)+B_{m}\left(\widehat{\Theta}^{\mathrm{T}}(t)-\Theta^{\mathrm{T}}\right) x(t)+B_{m}(\hat{\sigma}(t)-\sigma(t))
$$

As adaptation takes place, $\widehat{\Theta}(t) \rightarrow \Theta, \hat{\sigma}(t) \rightarrow \sigma$ and the aircraft dynamics in Eq. (7) approach those of the reference model in Eq. (3). The adaptive parameters are estimated based upon tracking error with respect to the MRAC reference models. The error for the roll axis is given in Eq. (8) and for the pitch axis in Eq. (9).

$$
\begin{gathered}
\tilde{x}_{p}(t)=p_{m}(t)-p(t) \\
\tilde{x}_{q}(t)=\left[\begin{array}{c}
\int q_{m}(t)-\int q(t) \\
q_{m}(t)-q(t)
\end{array}\right]
\end{gathered}
$$

Further detail on the design and implementation of the model reference adaptive controller is given in Ref. 6. In order to evaluate the effect of added complexity, three model reference adaptive controllers were evaluated, which are outlined below.

\section{SMRAC - Simplified MRAC}

Simple adaptive update laws for the roll and pitch axis adaptive parameters are given in Eqs. (10) and (11), respectively. The controller employing these update laws is referred to as the simplified MRAC, or sMRAC. The pitch axis adaptive law in Eq. (11) was further simplified by tuning the elements of $P_{q}$ to essentially eliminate the influence of the integral of the pitch tracking error $\int q_{m}(t)-\int q(t)$ on the adaptive parameter $\hat{\theta}_{q 2}$ and by tuning the learning rate $\Gamma_{q 1}$ to keep the adaptive parameter $\hat{\theta}_{q 1}$ small. This was done for all of the MRACs. In doing so, the pitch and roll axes were each left with a single adaptive parameter dependent upon a single input and a single error term.

$$
\begin{gathered}
\dot{\hat{\theta}}_{p}=\Gamma_{\mathrm{p}} \tilde{x}_{p} P_{p} B_{m_{p}} p \\
{\left[\begin{array}{c}
\dot{\hat{\theta}}_{q 1} \\
\dot{\hat{\theta}}_{q 2}
\end{array}\right]=\left[\begin{array}{cc}
\Gamma_{\mathrm{q} 1} & 0 \\
0 & \Gamma_{\mathrm{q} 2}
\end{array}\right] \tilde{x}_{q}^{T} P_{q} B_{m_{q}} x_{q}}
\end{gathered}
$$




\section{2. onMRAC - Optimal Control Modification and Normalization MRAC}

Another controller was designed by increasing the complexity of the sMRAC adaptive update laws to address the potential for over-adaptation, especially during high frequency maneuvers. The more complex update laws included optimal control modification $(\mathrm{OCM})^{7}$ and normalization of the learning rate. The OCM and normalization terms have tunable parameters $v$ and $R$ respectively. The resulting controller is referred to as onMRAC. With these two features added, the update law for the roll axis goes from Eq. (10) to Eq. (12). The pitch axis goes from Eq. (11) to Eq. (13).

$$
\begin{gathered}
\dot{\hat{\theta}}_{p}=\frac{\Gamma_{\mathrm{p}}}{1+R_{\theta_{p}} p^{2}}\left(\tilde{x}_{p} P_{p} B_{m_{p}} p-v_{\theta_{p}} p^{2} \widehat{\theta}_{p} B_{m_{p}}^{T} P_{p} A_{m_{p}}^{-1} B_{m_{p}}\right) \\
{\left[\begin{array}{c}
\dot{\hat{\theta}}_{q 1} \\
\dot{\hat{\theta}}_{q 2}
\end{array}\right]=\frac{\left[\begin{array}{cc}
\Gamma_{\mathrm{q} 1} & 0 \\
0 & \Gamma_{\mathrm{q} 2}
\end{array}\right]}{1+x_{q}^{T} R_{\theta_{q}} x_{q}}\left(\tilde{x}_{q}^{T} P_{q} B_{m_{q}} x_{q}-x_{q} v_{\theta_{q}} x_{q}^{T}\left[\begin{array}{c}
\hat{\theta}_{q_{1}} \\
\hat{\theta}_{q_{2}}
\end{array}\right] B_{m_{q}}^{T} P_{q} A_{m_{q}}^{-1} B_{m_{q}}\right)}
\end{gathered}
$$

\section{3. onMRAC+ - OCM, Normalization, and External Disturbance Term MRAC}

A third adaptive controller was created by adding complexity to the onMRAC design in the form of an additional adaptive parameter in both the pitch and roll axes, $\hat{\sigma}$. The additional adaptive parameters are matched to the time varying disturbance term in both the pitch and roll axes. ${ }^{6}$ This term will adapt to coupling effects between the axes. The update laws for the roll and pitch axis time varying disturbance adaptive terms are given in Eqs. (14) and (15), respectively. The controller incorporating these parameters is referred to as onMRAC+.

$$
\begin{gathered}
\dot{\hat{\sigma}}_{p}=\frac{\Gamma_{\sigma_{\mathrm{p}}}}{1+R_{\sigma_{p}} p^{2}}\left(\tilde{x}_{p} P_{p} B_{m_{p}}-v_{\theta_{p}} \hat{\sigma}_{p} B_{m_{p}}^{T} P_{p} A_{m_{p}}^{-1} B_{m_{p}}\right) \\
\dot{\hat{\sigma}}_{q}=\frac{\Gamma_{\sigma_{\mathrm{q}}}}{1+x_{q}^{T} R_{\sigma_{q}} x_{q}} \tilde{x}_{q}^{T} P_{q} B_{m_{q}}
\end{gathered}
$$

\section{Failure Conditions}

Two failure scenarios were implemented in order to evaluate the ability of the various controllers to adapt to undesired coupling effects between the pitch and roll axes. Testing under other failure conditions are presented in a companion paper given in Ref. 8; these failures include a pitch damping reduction and a roll damping reduction.

\section{A. Roll to Pitch Input Coupling}

An induced roll due to pitch input scenario was used to evaluate the adaptive controllers. This scenario simulates the coupling that may exist after a failure has occurred. An addition was made to each controller that added the lateral stick input to the longitudinal stick command that is fed into the NDI controller's reference model, but not into the MRAC's reference model. Figure 3 shows a pitch doublet followed by a roll doublet with this failure in flight for the baseline NDI controller. The effect of the coupling can be seen where pitch rate is generated during the roll doublet. Roll-to-pitch coupling was specifically chosen to degrade the handling qualities of the aircraft based upon a study conducted by the United States Air Force (USAF) Test Pilot School (Edwards Air Force Base, Edwards, California), Project Icarus, ${ }^{9}$ which found that the coupling of pitch due to roll degrades handling qualities three times more than an equivalent amount of roll due to pitch. 


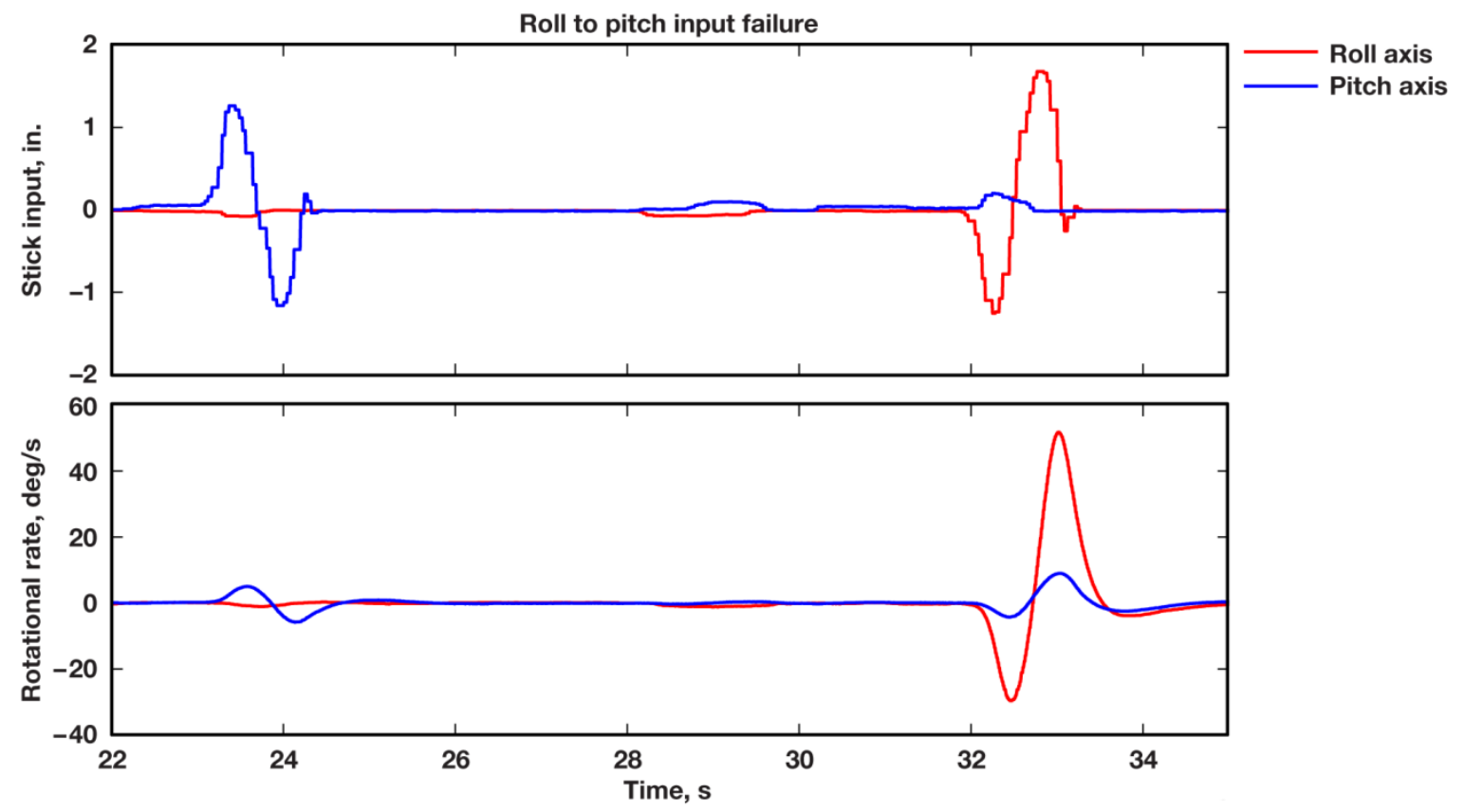

Figure 3. Roll to pitch input failure doublet response with NDI controller in flight.

\section{B. Left Stabilator Failure}

The adaptive controllers were also evaluated for a failure in which the left stabilator was frozen at a deflection angle of zero degrees which is slightly off trim. This failure models a situation where there is a mechanical failure that prevents movement of a primary control surface. The stabilators are used for both pitch and roll control so when the pilot attempts either lateral or longitudinal control, the aircraft will exhibit a cross coupling effect. Flight data for the baseline NDI controller is shown in Fig. 4 where the pilot performed a pitch doublet and a roll doublet with this failure. The most noticeable effect is the added roll rate during a pitch doublet.

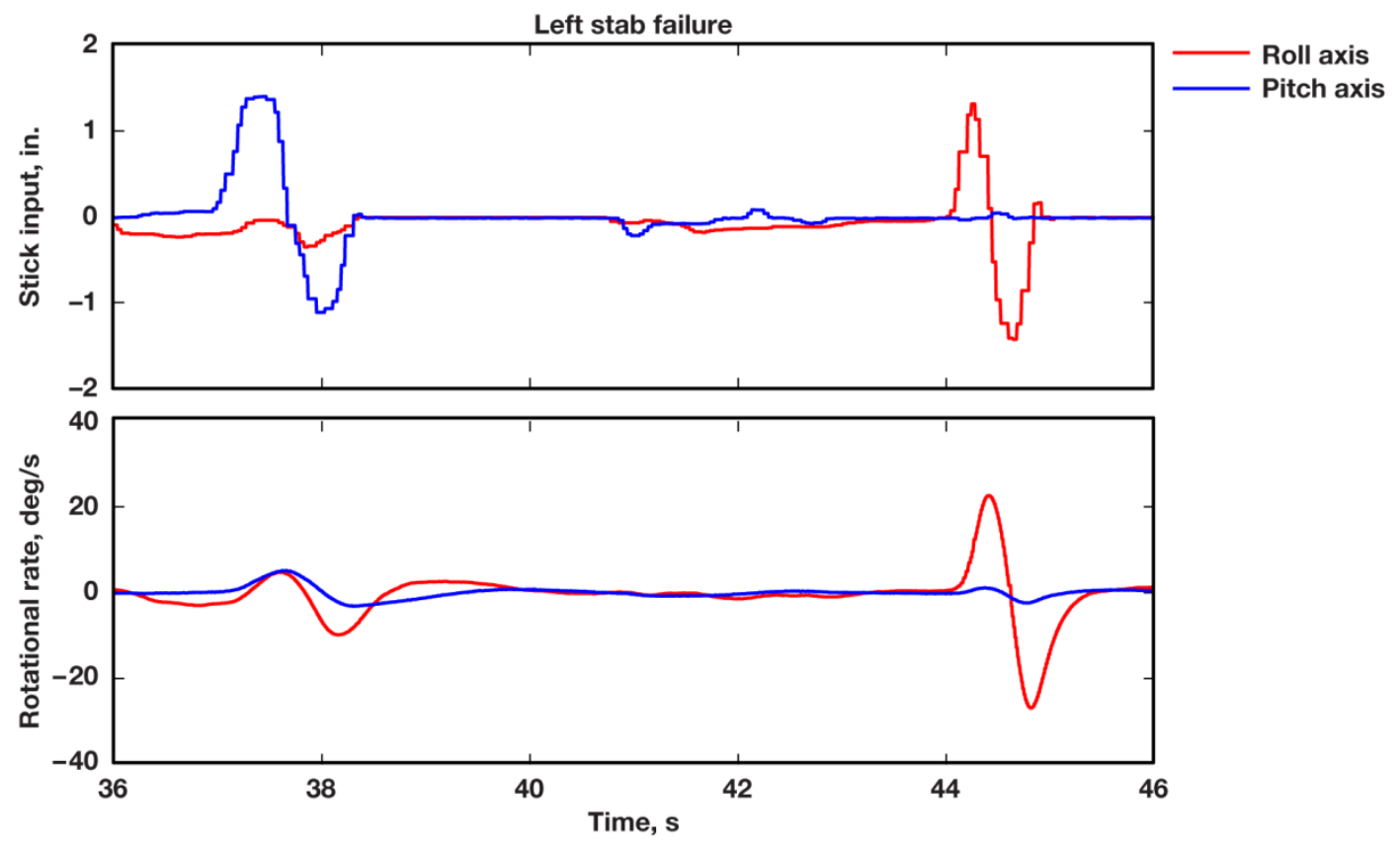

Figure 4. Left stab failure doublet response with NDI controller in flight.

American Institute of Aeronautics and Astronautics 


\section{Metrics and Evaluation Methods}

Several metrics are used to measure how well the adaptive controllers reduce the effects of the failures. These metrics range from measuring the tracking error between the MRAC reference model and the feedback to handling qualities ratings given by a pilot. Also, criteria for evaluating the handling qualities based on the magnitude of coupling is investigated.

\section{A. MRAC Tracking Error Metric}

The extent to which the adaptive controllers bring the response of the aircraft back towards the MRAC reference model is measured using a tracking error metric. Equation (16) is used to compute the tracking error as a function of time during the maneuvers to compare the reference model dynamics to the actual dynamics of the aircraft.

$$
\text { MRAC Tracking Error Metric }(t)=\sqrt{\int_{t_{0}}^{t}\left(x_{m}(\tau)-x(\tau)\right)^{2} d \tau}
$$

\section{B. Stick Position Trace}

Another metric is to examine the trace of the stick position during a maneuver. This plot of pitch-stick input versus roll-stick input will show the general trend in compensation that the pilot is using. The pilot is also an adaptive controller, and it is important to see how he/she is adapting to the failure as well as the controller.

\section{Cooper-Harper Ratings / Handling Qualities Tasks}

The degree to which tracking error must be reduced to have acceptable performance to the pilot is unknown. In-flight handling qualities ratings contribute to a more comprehensive and complete evaluation of how well the controller is performing. The adaptive controllers may help reduce coupling between the axes and improve tracking performance, but at the same time may degrade other aspects of the handling qualities of the aircraft. To achieve an overall assessment of the controller, handling qualities evaluations were conducted with multiple pilots. The roll to pitch input coupling failure was evaluated with three pilots, and the left stabilator failure was evaluated with two pilots. The handling qualities of the aircraft can then be quantified using the Cooper-Harper $(\mathrm{CH})$ and pilot induced oscillation (PIO) rating scales. ${ }^{10,11}$ The pilots also gave comments after each task describing the whole system and any unusual or objectionable aspects of the controller. A more general assessment of how well the controller is doing can be made by examining both, the overall trends from the $\mathrm{CH}$ and PIO ratings, and the comments from the pilots.

Handling qualities evaluations require well-defined tasks with specific desired and adequate criteria outlined. ${ }^{10}$ A set of tasks was developed that incorporated both the pitch and roll axes to excite any coupling that may exist. The tasks that were developed involved having the test aircraft fly behind and within close proximity to a target aircraft. The test aircraft would acquire and track points on the target aircraft. These tasks can relate to real world tasks a pilot may face after a failure has occurred such as air-to-air refueling in order to get back to an airport, or once at an airport, tracking an instrument landing system (ILS) for landing. The lateral-directional aspect of this type of tracking task has been shown to correlate well to tracking during offset landings. ${ }^{12}$ The task is chosen to have the pilot in-the-loop with the controller to uncover anything open loop control may not reveal.

The setup for the tasks involved having the test aircraft fly in trail behind a target aircraft. To get into this position, a piece of tape placed on the canopy of the test aircraft was aligned with the tail hook of the target aircraft in front, as shown in Fig. 5, to ensure a consistent starting geometry for each test. The vertical spacing at this position was sufficient to avoid effects from the jet wash. The horizontal spacing was set at two ship lengths (one ship length is $56 \mathrm{ft}$ ). From the perspective of the pilot in the test aircraft, two ship lengths place the wingspan of the lead aircraft to be about the same size as the rearview mirror. Once in this position, the pilot selects the failure on the flight computer. The failure is initiated by the pilot pressing the nose wheel steering button. Next the MRAC is turned on by another press of the nose wheel steering button. The pilot then begins the first task. This task is a gross acquisition task, where the pilot of the test aircraft maneuvers the plane to line up a reference point on the heads-up display (HUD) with the wingtip of the target aircraft while maintaining the 2-ship length horizontal spacing. Figure 6 shows the ending reference point for this task. The task was repeated by going to both sides because the failures are asymmetric so an assessment in both directions is necessary. 


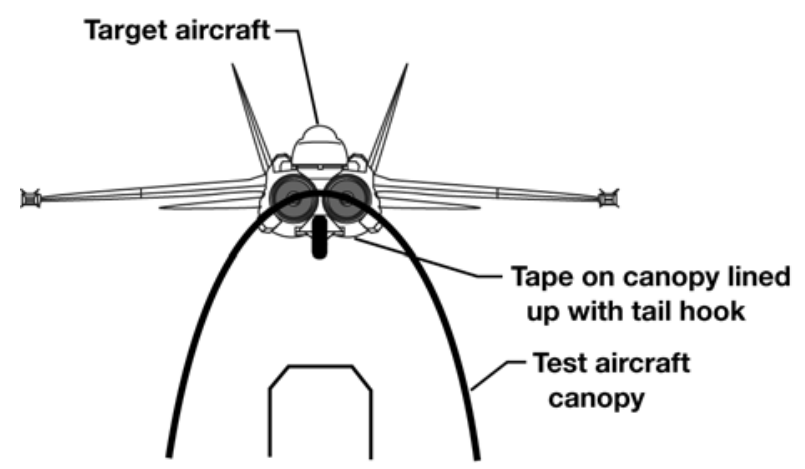

Figure 5. Gross acquisition starting point (not to scale).

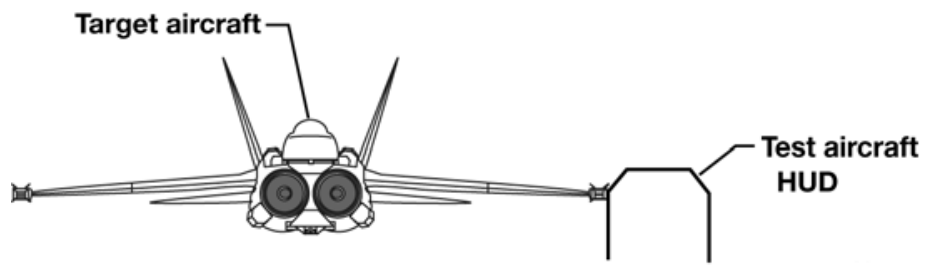

Figure 6. Gross acquisition ending point/fine tracking reference point (not to scale).

Following gross acquisition of the wingtip, the pilot initiated the fine tracking task. The pilot was tasked with holding the HUD reference point on to the wingtip for at least 10 to $15 \mathrm{~s}$. Figure 7 shows an image taken from the back seat of the target aircraft and an image taken from the HUD of the test aircraft during the fine tracking task.

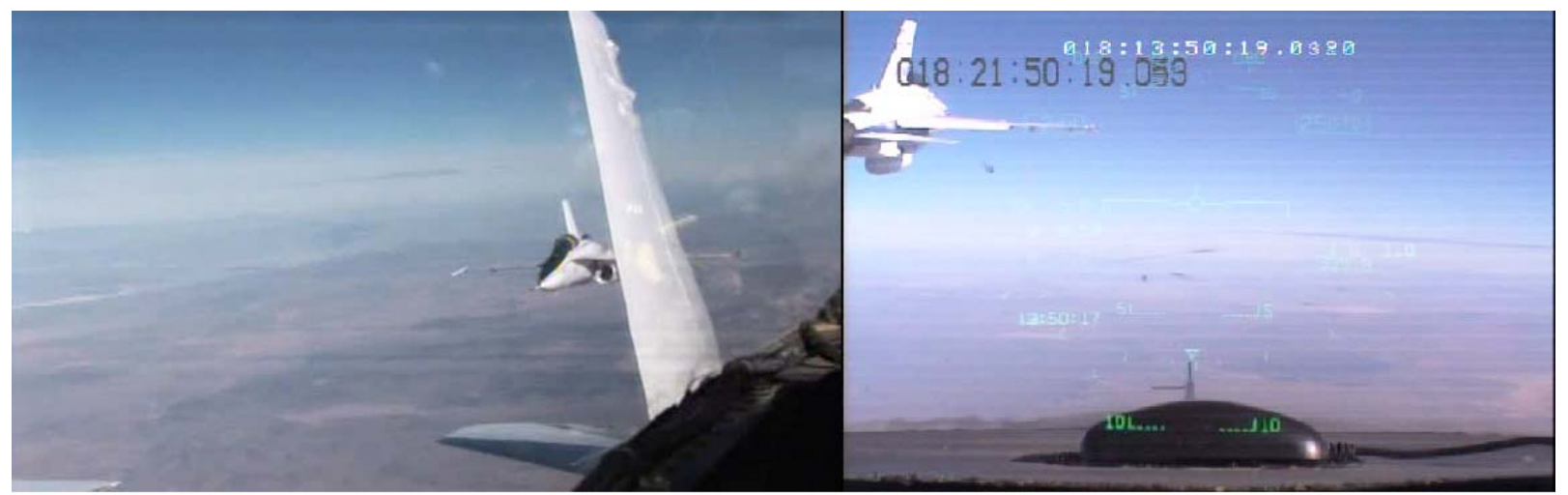

Figure 7. View from each aircraft during the tracking task.

For the $\mathrm{CH}$ ratings, desired and adequate performance was defined for both tasks. For the gross acquisition task, one or no overshoots was defined as desired and 2 overshoots was adequate. For the fine tracking task, keeping the bracket of the HUD on the wingtip for $80 \%$ of the time was desired and $50 \%$ was adequate.

\section{A. Inter-axis Coupling Handling Qualities Criteria}

The severity of the failure and the resulting magnitude of coupling have a correlation to the handling qualities ratings given by a pilot. ${ }^{9}$ Since coupling of the pitch and roll axes is much more common in helicopters than fixed wing aircraft, a handling qualities criteria based on the magnitude of coupling exists in ADS-33E ${ }^{13}$ which defines handling qualities for helicopters. An accepted criterion for fixed wing aircraft does not exist. In 2010, the USAF Test Pilot School used an F-16 aircraft (Lockheed Martin Corporation, Bethesda, Maryland) with induced coupling to evaluate similar criteria for fixed-wing aircraft, Project Icarus. ${ }^{9}$ Both the helicopter and proposed fixed wing 
criteria, shown in Fig. 8, are used to relate the metric of the degree of coupling between the two axes to handling qualities levels.

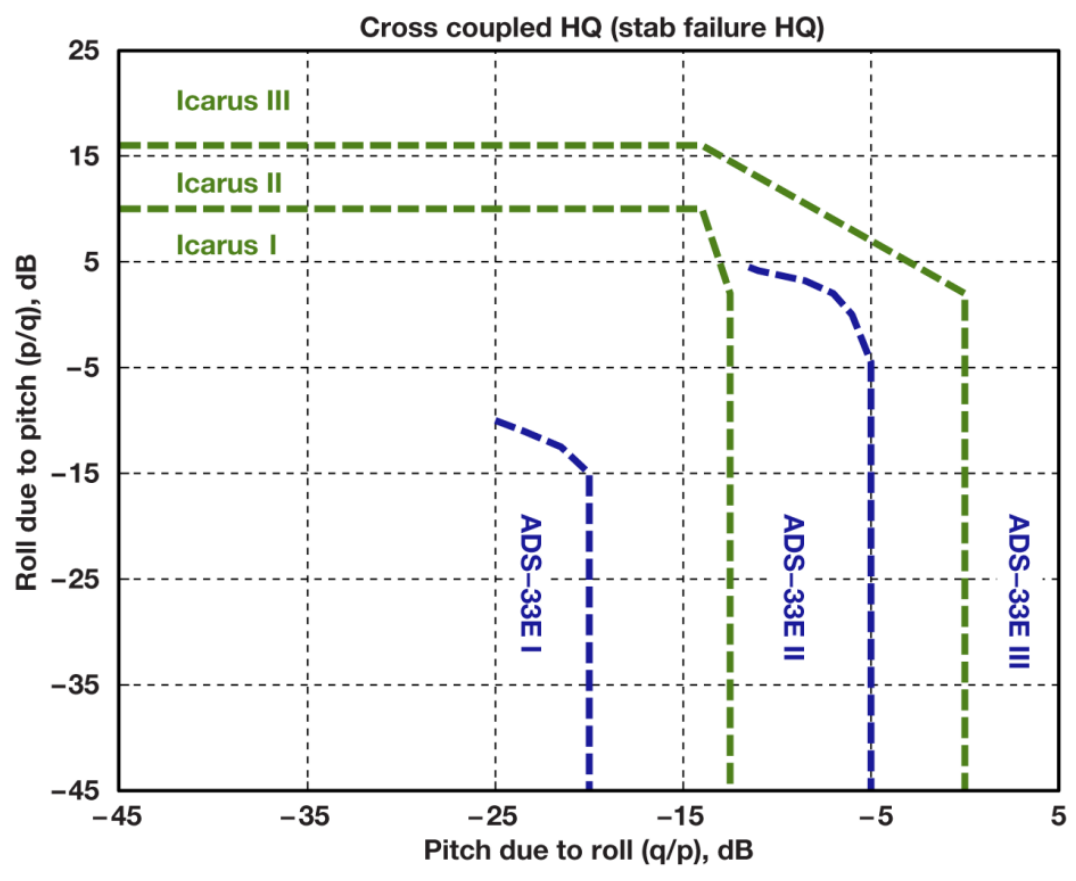

Figure 8. Cross coupling handling qualities criteria.

To apply the cross coupling handling qualities criteria, the magnitude of coupling must be calculated. The criteria were developed by taking the average ratio of coupling over a specific range of frequencies determined by the bandwidth and the phase cross over frequencies. Frequency responses were obtained using frequency sweeps. A lesson learned during Project Icarus, was that the calculated coupling values were "very sensitive to small changes throughout the frequency analysis." "Another note was that the frequencies over which the magnitude of coupling was averaged were not always indicative of the frequencies of the inputs used by the pilots during a given task. ${ }^{9} \mathrm{~A}$ difficulty in applying this technique to nonlinear, time-varying adaptive controllers is that adaptive parameters during a frequency sweep may not have the same values as during a given task for a pilot. Also, the adaptive parameters may grow during a frequency sweep, and freezing them at a set value may not capture the actual dynamics involved as the adaptive parameters often move with inputs, such as $\hat{\sigma}$, the term matched to time varying disturbances. For these reasons a time domain approach was used to compute the average magnitude of coupling observed during a specific task.

The magnitude of coupling for the baseline aircraft was assumed to be negligible $(<-40 \mathrm{~dB})$. The roll to pitch input coupling failure adds only pitch due to roll. In order to calculate the magnitude of this coupling, the signals of the controller were reconstructed, and the amount of pitch response due only to the actual longitudinal stick input could be calculated and subtracted from the pitch response of the aircraft. The result is the pitch rate due to both roll rate and the current state of the adaptive parameters, thus removing any effect from the pilot's longitudinal inputs. This remaining parameter is the effective coupling of roll into pitch. This value is plotted along with roll rate in Fig. 9 over the span of a fine tracking task. The magnitude of coupling was then calculated by taking the ratio between these two parameters at peak values during the span of the task. This ratio varies during any specific input depending on the frequency of the input so the point value used for the coupling metric must be taken as the average over the span of the task. This value then represents the average amount of coupling the pilot experienced during the task. 


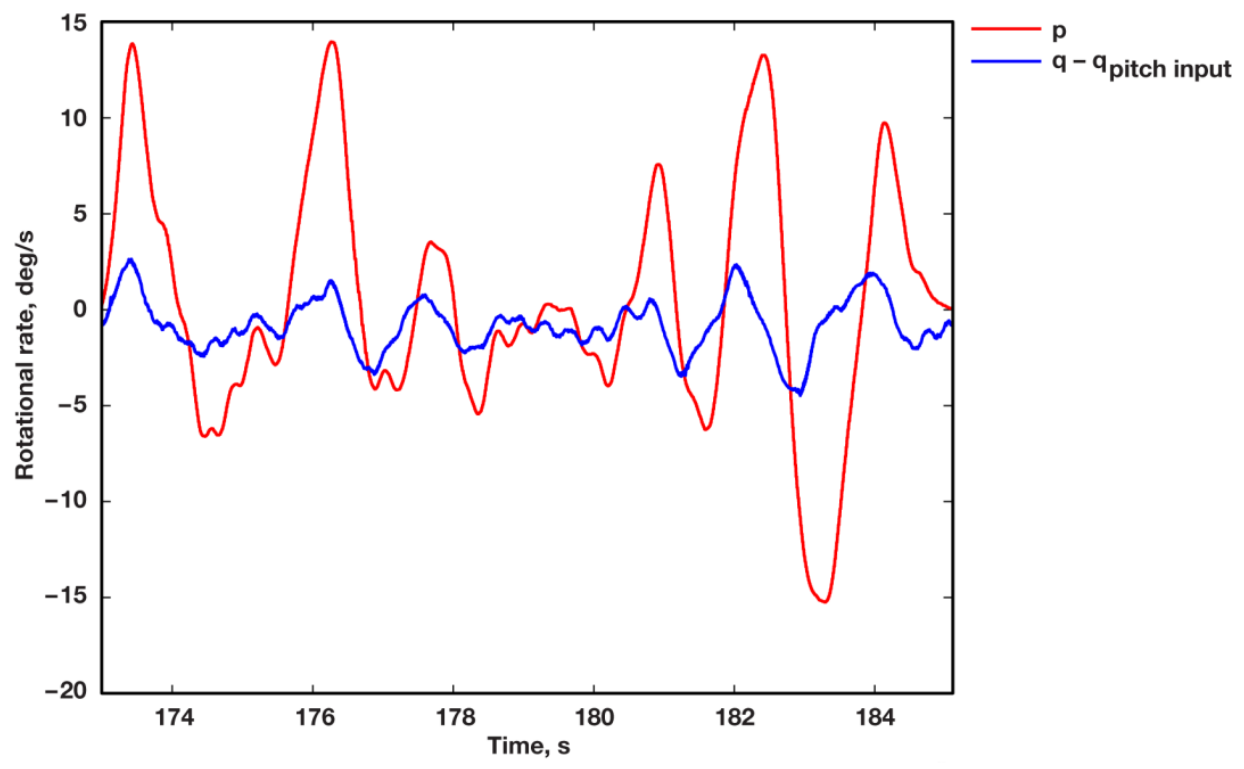

Figure 9. Example plot of pitch rate due to roll rate coupling for roll to pitch input coupling failure during a fine tracking task.

\section{Flight Results}

Flight tests were carried out to evaluate the different controller's performance with the two failures. All of the tasks were performed at Mach 0.58 and 25,000 ft.

\section{A. Roll to Pitch Input Coupling Failure Results}

Three test pilots flew the NDI and the three MRACs with the roll to pitch input coupling failure and also the baseline NDI without a failure for comparison. Figure 10 shows both the $\mathrm{CH}$ and PIO ratings given by the pilots for the gross acquisition task. For the $\mathrm{CH}$ ratings for this task, every pilot gave Level 2 handling qualities for the baseline aircraft with the failure. With the MRAC turned on, handling qualities either remained Level 2 or improved to Level 1 for all pilots.
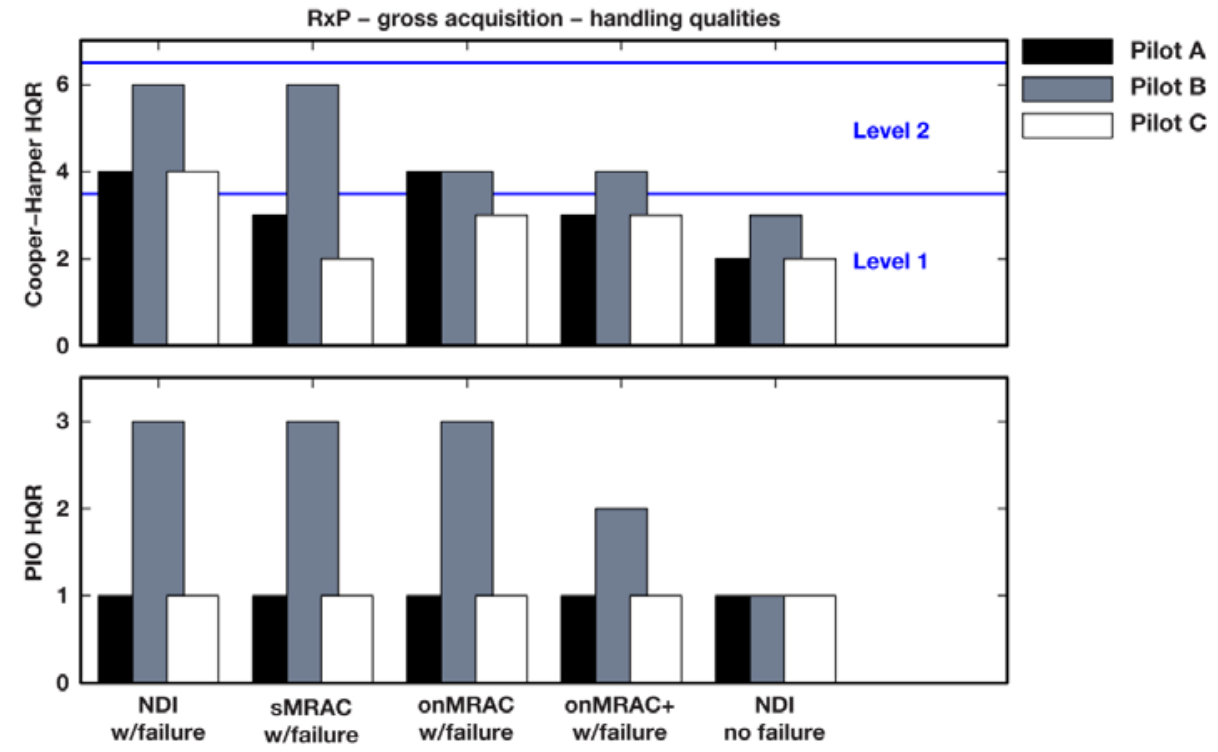

Figure 10. Roll to pitch input failure gross acquisition handling qualities. 
Pilot A and Pilot C show similar trends. They both gave PIO ratings of one, which indicates no undesired motion was noticed for any configuration even though coupling between the axes was present. Even with the baseline NDI with the failure, desired performance could be achieved. Based on the $\mathrm{CH}$ ratings given, for the sMRAC and onMRAC+ the workload required to perform the task was reduced and the onMRAC showed either equal workload or improved workload from that of the baseline controller with the failure. Overall the MRAC either improved the $\mathrm{CH}$ and $\mathrm{PIO}$ ratings or they remained the same.

Pilot B is an important case because he rated the failure as having undesirable motion that affected his ability to perform the task and specifically mentioned that he felt coupling; the other pilots often stated they observed no coupling. Pilot B also gave the failure the worst $\mathrm{CH}$ rating of 6 for the baseline NDI controller indicating he was most affected by this failure. With the sMRAC, he was still getting just adequate performance with the same level of workload. With the onMRAC, however, he saw improvement and was able to get desired performance, but the undesirable motion was still a factor in performing the task. With the onMRAC+ this was no longer the case and he gave a PIO rating of 2 meaning the task performance was no longer compromised by the failure. For onMRAC+, his PIO rating and $\mathrm{CH}$ rating are now both one point from what he gave for the baseline controller with no failure, showing considerable improvement in reducing the effect of the failure.

Figure 11 shows the magnitude of coupling of pitch rate due to roll rate for each pilot and configuration. This was calculated during the gross acquisition task. The value was averaged over this task as adaptation was occurring. For each pilot, the magnitude of coupling is seen to generally decrease as complexity is added to the system. Differences between the various pilot's gain and frequency of inputs during the task results in different magnitudes of coupling. For example, Pilot $\mathrm{C}$ has a much higher gain piloting technique than the other three and performs the gross acquisition task far more aggressively, which showed a lower amount of coupling response. This variation in amount of coupling is due to the fact that for the failure, roll stick is added onto pitch stick, which then goes through the pitch reference model so the amount of coupling is frequency dependent based upon the relationship between the pitch and roll reference models. Pilot $\mathrm{C}$ also would move the stick around prior to starting the task to get a feel for the aircraft which would cause slight adaptation prior to the task.

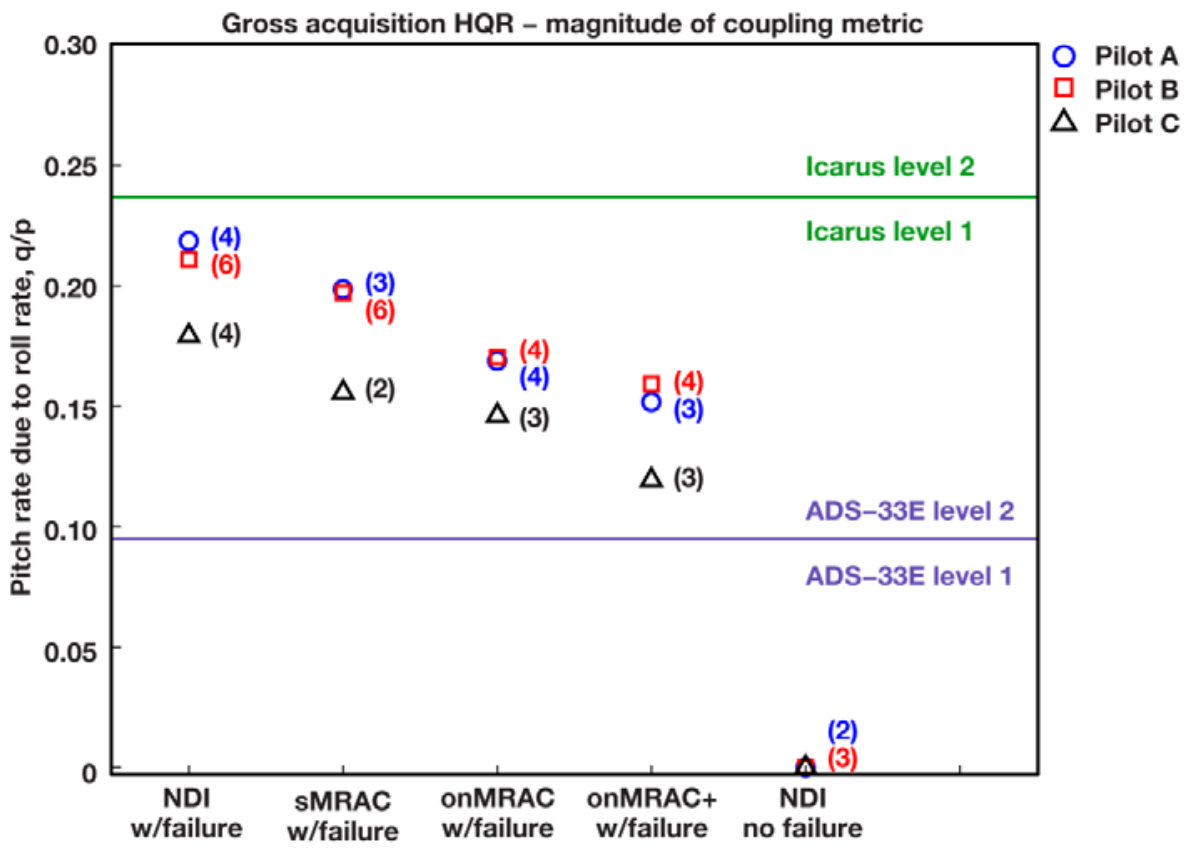

Figure 11. Gross acquisition magnitude of coupling.

Figure 12 shows the handling qualities ratings given for the fine tracking task. For both $\mathrm{CH}$ and PIO ratings, the most complex MRAC, onMRAC+, with the failure got the same ratings as the baseline NDI with no failures. Based on $\mathrm{CH}$ and PIO ratings, it has effectively removed the failure. Pilot comments indicate that the workload was seen to decrease for the MRACs. For all pilots, as complexity is incrementally increased, the $\mathrm{CH}$ ratings either stay the same or decrease, showing steady improvement. Based on the PIO scale, no pilot saw any PIOs; however, undesirable motions were observed. The most undesirable motion was observed for the NDI with the failure. Both Pilot B and 
Pilot $\mathrm{C}$ noticed this for the NDI with the failure case. On tests with the three MRACs, they felt there were no undesirable motions.

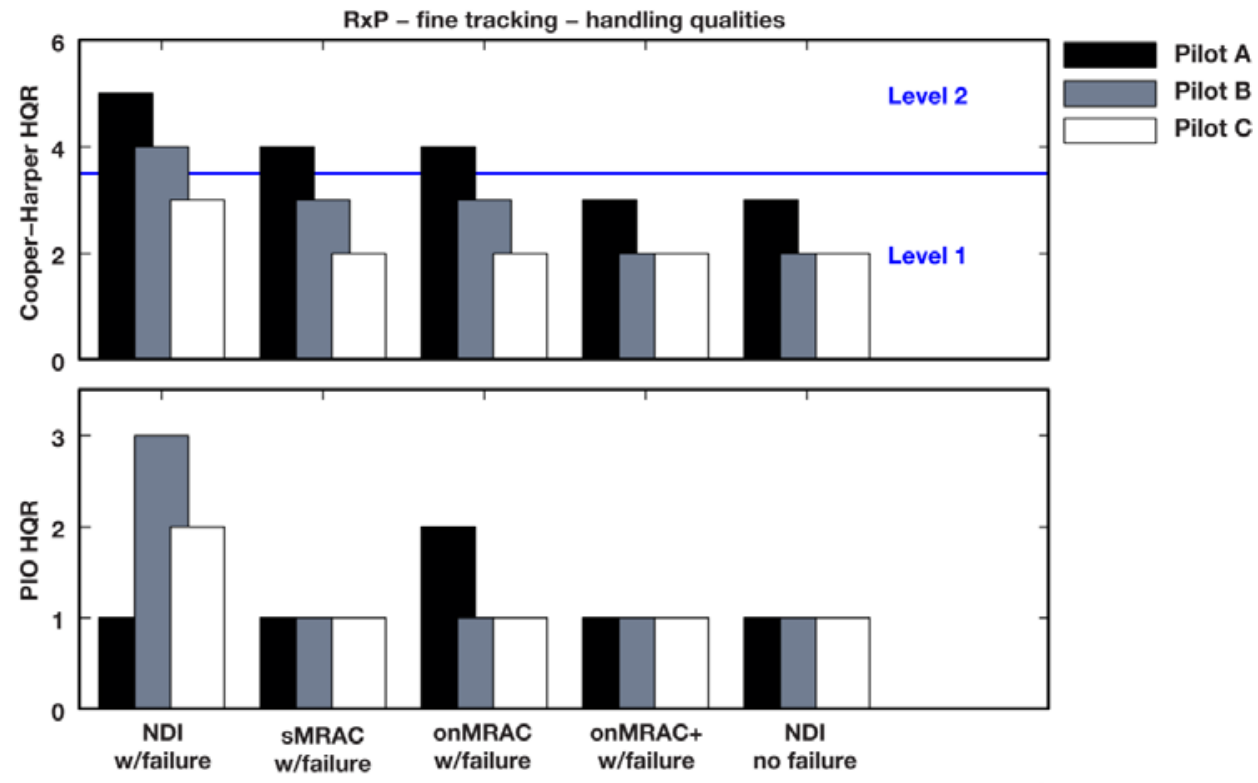

Figure 12. Roll to pitch input failure fine tracking handling qualities.

Figure 13 shows that the magnitude of coupling is seen to be reduced as controller complexity is added. Figure 13 also shows a correlation between the amount of coupling the pilot experienced and the $\mathrm{CH}$ ratings given, with both showing improvement with added complexity. The two criteria that are shown in Fig. 13, Icarus and ADS-33E, vary significantly; however, from the data collected from these three pilots, both criteria may apply depending on the pilot. For Pilot $\mathrm{C}$, the ratings match closer to the Icarus line while Pilot A lies closer to ADS-33E. Depending on the pilot, the $\mathrm{CH}$ ratings may trend closer to the criteria derived from the F-16 aircraft or towards the helicopter derived criteria.

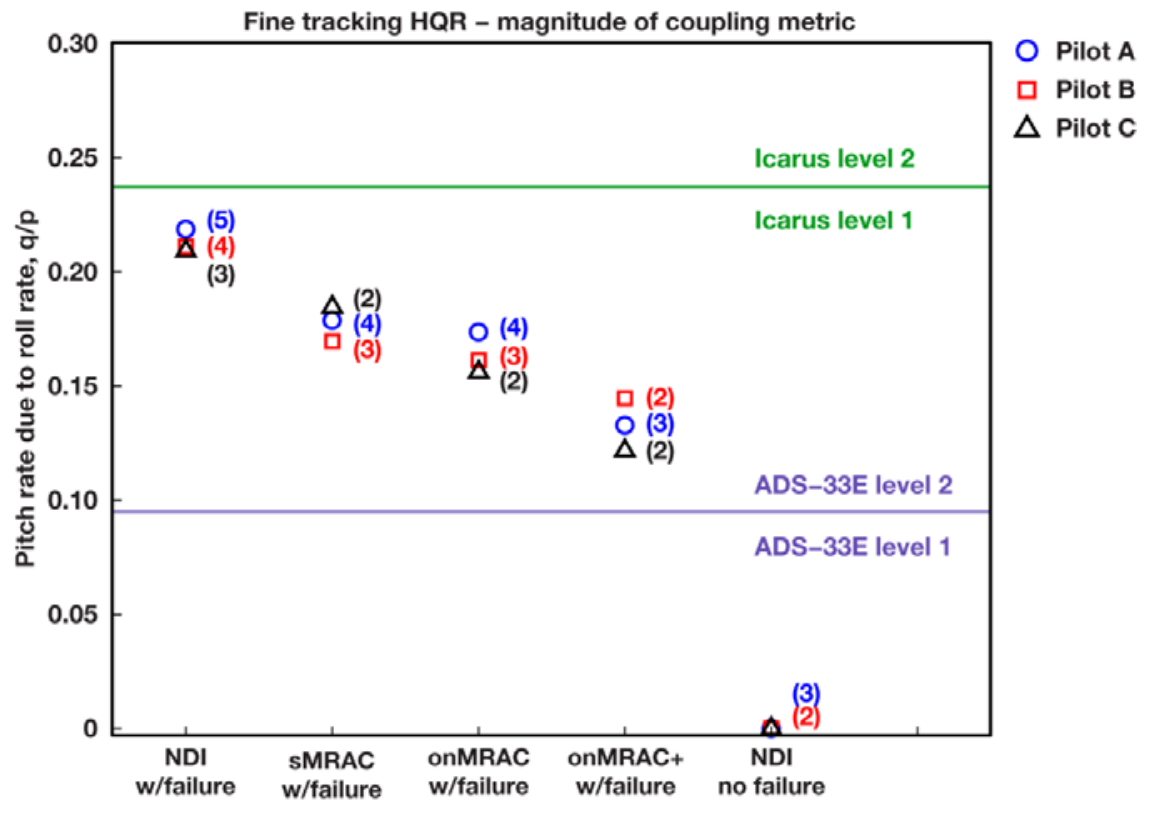

Figure 13. Fine tracking magnitude of coupling.

American Institute of Aeronautics and Astronautics 
To examine what is occurring in more detail, data from Pilot A during the fine tracking task is investigated. Figure 14 shows the traces of Pilot A's input during this task while behind the left wing of the lead aircraft for each controller configuration. The red line drawn is a linear fit to the average pitch-stick input for a given roll stick input. The pilot has a steady offset of about 0.2 in roll stick due to the downwash effect from the lead aircraft resulting in a steady roll that the pilot must compensate for. Since the failure adds roll stick to pitch stick, a resulting opposite sign pitch stick must be applied by the pilot that would increase in magnitude as roll stick increases. Thus, the slope of the red line relates to the compensation that the pilot is applying to counter the coupling of the roll axis into pitch. The slope of this line is nearly halved for the onMRAC+ case from that of the NDI. Only slight changes from the NDI are seen for the sMRAC and onMRAC configurations. All three pilots showed this same trend in pilot compensation. This trend in pilot compensation also correlates to the $\mathrm{CH}$ ratings given indicating the task became easier to perform, and less workload was required since less coupling had to be compensated for by the pilot.
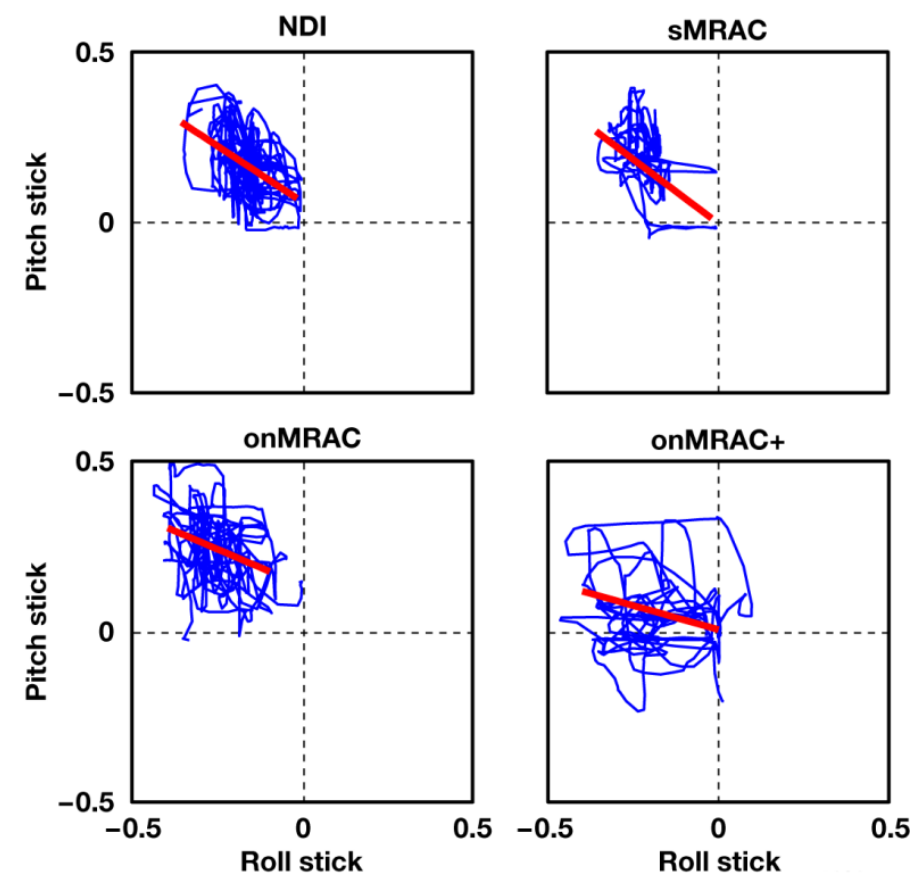

Figure 14. Input trace from Pilot A during fine tracking tasks.

The tracking error metric during this task is shown in Fig. 15 for the same pilot (A). The plot shows a large steady improvement for the onMRAC+ case with varied performance for the onMRAC and SMRAC in relation to the NDI. In the pitch axis, the sMRAC and onMRAC have only matched adaptive parameters and no term to deal with external disturbances such as error driven by variables in the roll axis. The only effective adaptive parameter in pitch for sMRAC and onMRAC is the $\hat{\theta}_{q 2}$ term which is related to pitch damping. If this parameter decreases, it will reduce the off axis pitch due to roll by reducing how much pitch response is generated by the input. This reduced pitch effectiveness has a limited effect at reducing the coupling. Whenever the pilot attempts to do a pitch maneuver, error will be generated due to the system now being over damped, and thus drive this parameter back towards its ideal level of zero for this failure. This effect can be seen in the tracking error as it will improve or worsen depending on how much roll versus how much pitch is being commanded as roll commands will drive it negative and pitch commands will drive it back towards zero. This variation in pitch damping is the reason that the tracking error for both sMRAC and onMRAC is showing little to no improvement from the NDI; however, this effect does have an effect on reducing coupling and thus improves handling qualities slightly. 


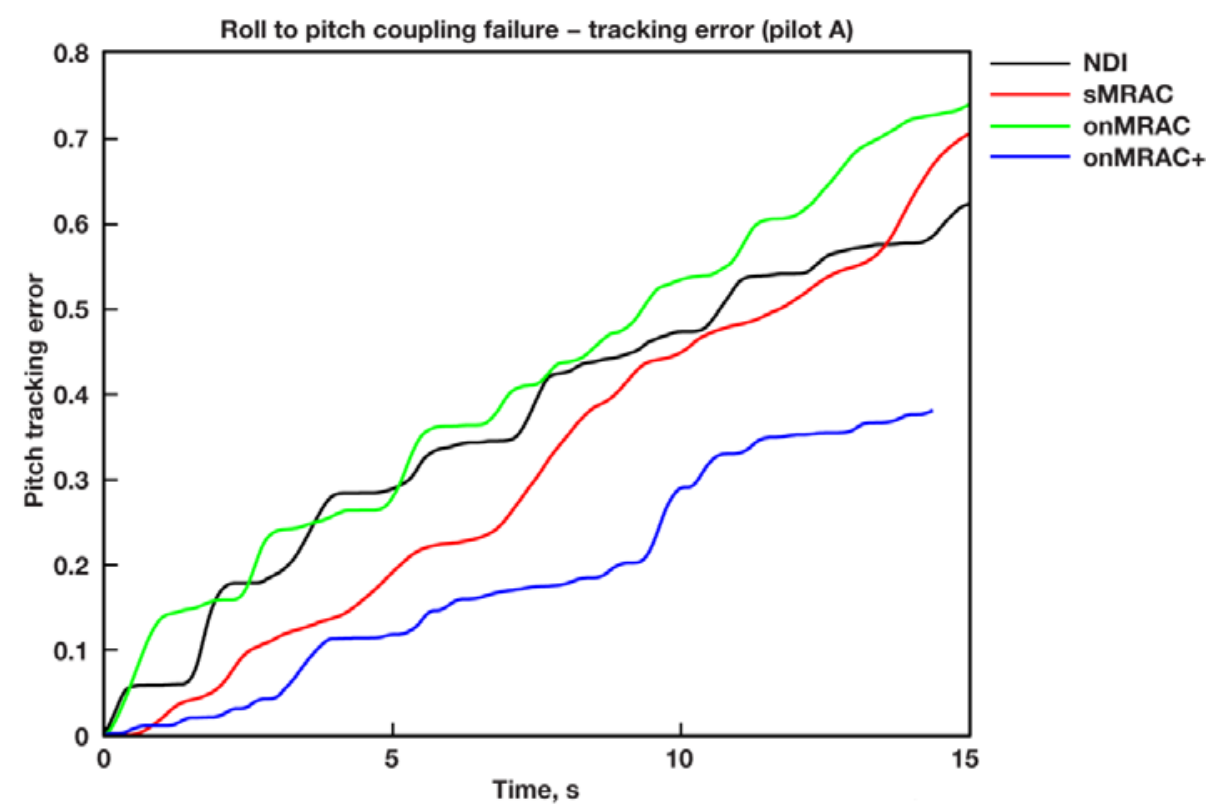

Figure 15. Pitch tracking error metric during fine tracking tasks.

For the onMRAC + case, there is the exogenous disturbance adaptive term $\left(\hat{\sigma}_{q}\right)$, which adapts to time varying disturbance error such as pitch error due to roll. The $\hat{\sigma}_{q}$ term is effectively a pitch acceleration command going into the NDI. If the $\hat{\sigma}_{q}$ term is removing the coupling created by the failure, the ideal value for $\hat{\sigma}_{q}$ would be equal but opposite in sign of $\dot{q}_{c m d}$ from the pitch reference model when fed with roll-stick input. This would eliminate the effects of the failure. Figure 16 shows the correlation between $\hat{\sigma}_{q}$ and $\dot{q}_{c m d}$ from roll stick input. In this figure $\dot{q}_{c m d}$ is reversed sign for better comparison. The two parameters do not track perfectly because there are other sources of error other than just that from roll stick being added to pitch stick, such as the other pitch adaptive parameters adapting undesirably in pitch creating error during pitch maneuvers. The two parameters do, however, track fairly well and the result is that it reduces the effect of roll stick being added into the pitch axis, effectively reducing the coupling.

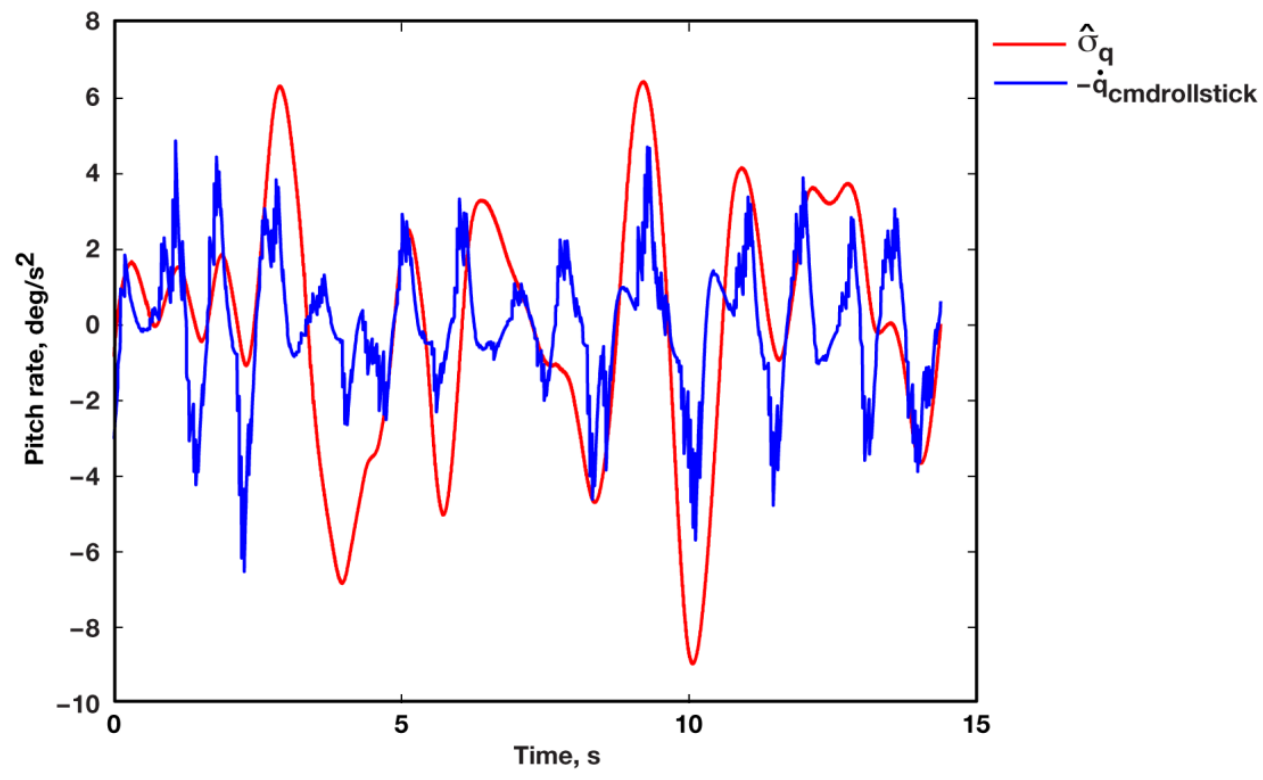

Figure 16. $\widehat{\sigma}_{q}$ and $\dot{q}_{c m d}$ from roll stick during fine tracking task.

American Institute of Aeronautics and Astronautics 


\section{A. Left Stabilator Failure Results}

Two pilots $(\mathrm{C}$ and $\mathrm{D})$ flew the handling qualities tasks with the left stabilator frozen at zero deflection angle, a slight offset from trim. For the gross acquisition task, both pilots rated the failure with the NDI and the three MRAC configurations within Level 2 showing no improvement. Figure 17 shows the ratings given for the fine tracking task, which shows borderline Level 1-Level 2 ratings. Pilot $\mathrm{D}$ gave every configuration the exact same rating for both $\mathrm{CH}$ and PIO. Pilot $\mathrm{C}$ observed some improvement with the sMRAC in both $\mathrm{CH}$ and PIO ratings. With the optimal control modification term and normalization, that improvement was removed, and the pilot cited increased workload as the cause. The failure condition itself was not a severe failure with the baseline controller. For the fine tracking task, the pilots were able to achieve desired performance with minimal to moderate compensation with just the NDI controller.

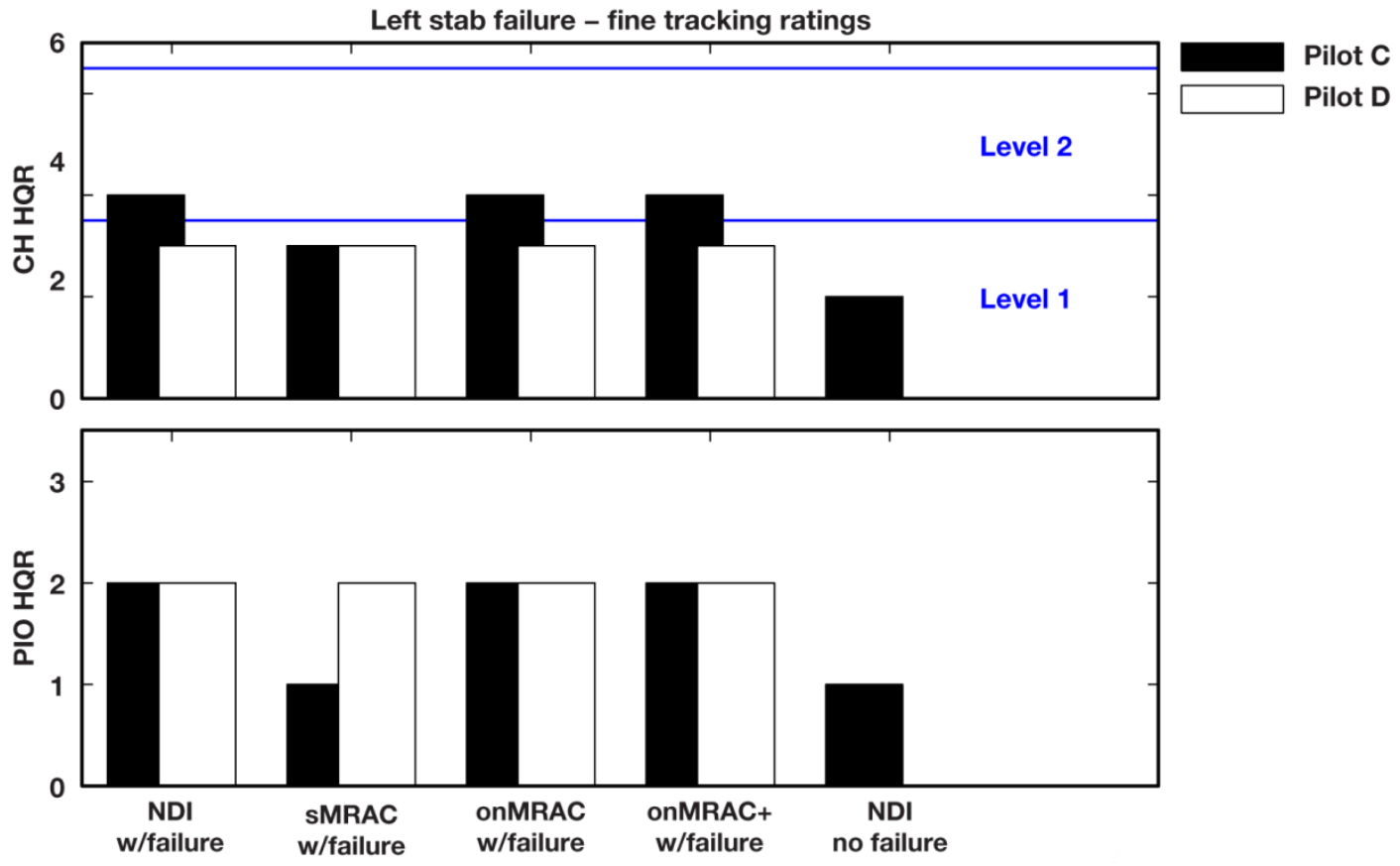

Figure 17. Left stab failure handling quality ratings.

Both pilots commented about poor predictability and sluggishness in pitch for the baseline NDI controller with the failure. Pilot C's comments on the MRAC configurations indicate that the predictability was still poor; however, Pilot D said the opposite, and that the onMRAC+ was predictable and not sluggish. He said it felt like a "normal Hornet (F/A-18)" except for sensitivity in pitch.

Figure 18 shows the tracking error metrics applied to the roll axis for Pilot $\mathrm{C}$ for each of the configurations. The three MRACs greatly reduced the tracking error in the roll axis. 


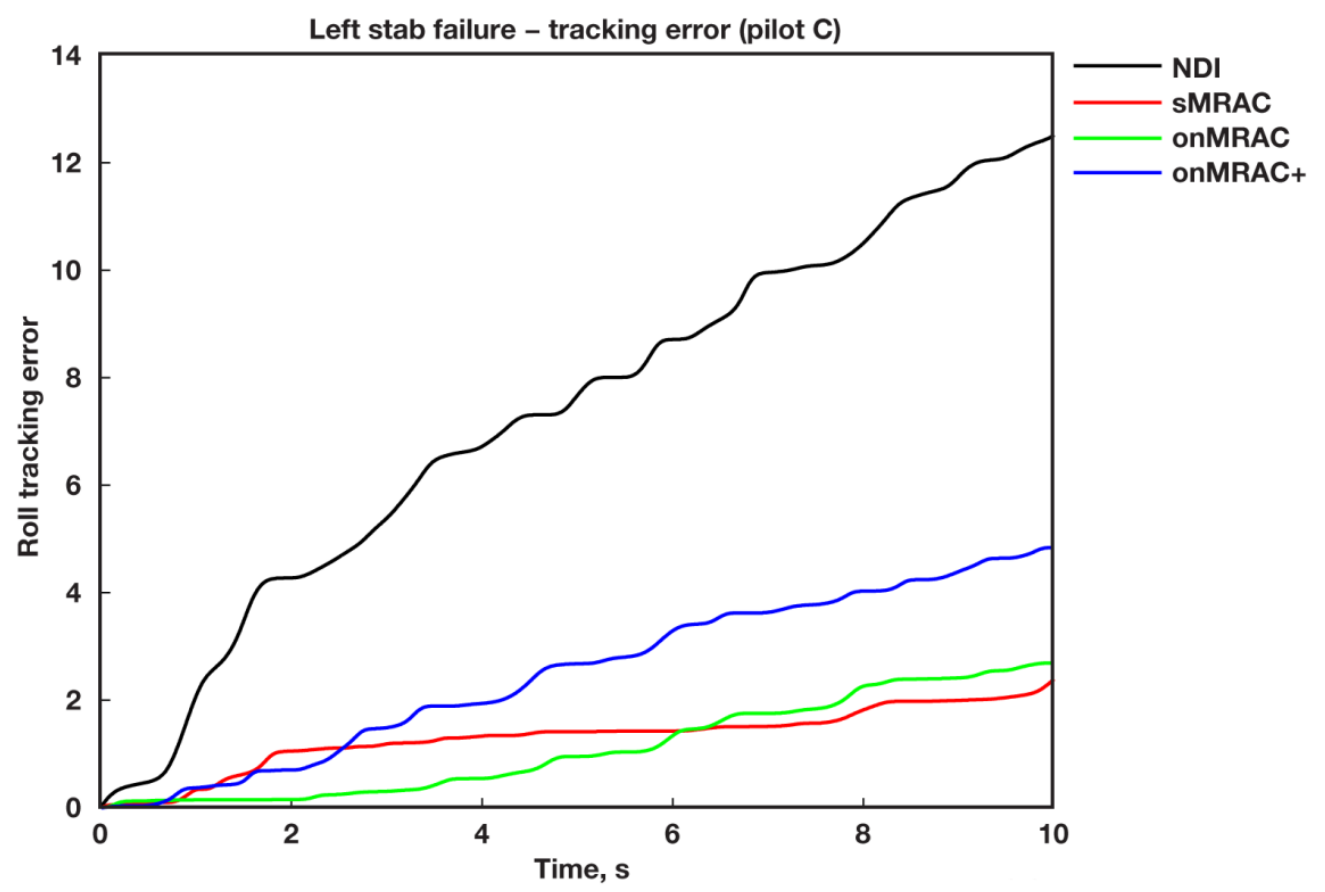

Figure 18. Roll tracking error for Pilot C.

Figure 19 shows the traces of Pilot C's input during the tracking tasks for each configuration. The NDI is showing the most lateral stick input, which matches the tracking error in that axis as it had the largest error. The onMRAC stick trace is showing mostly only a pitching input motion.
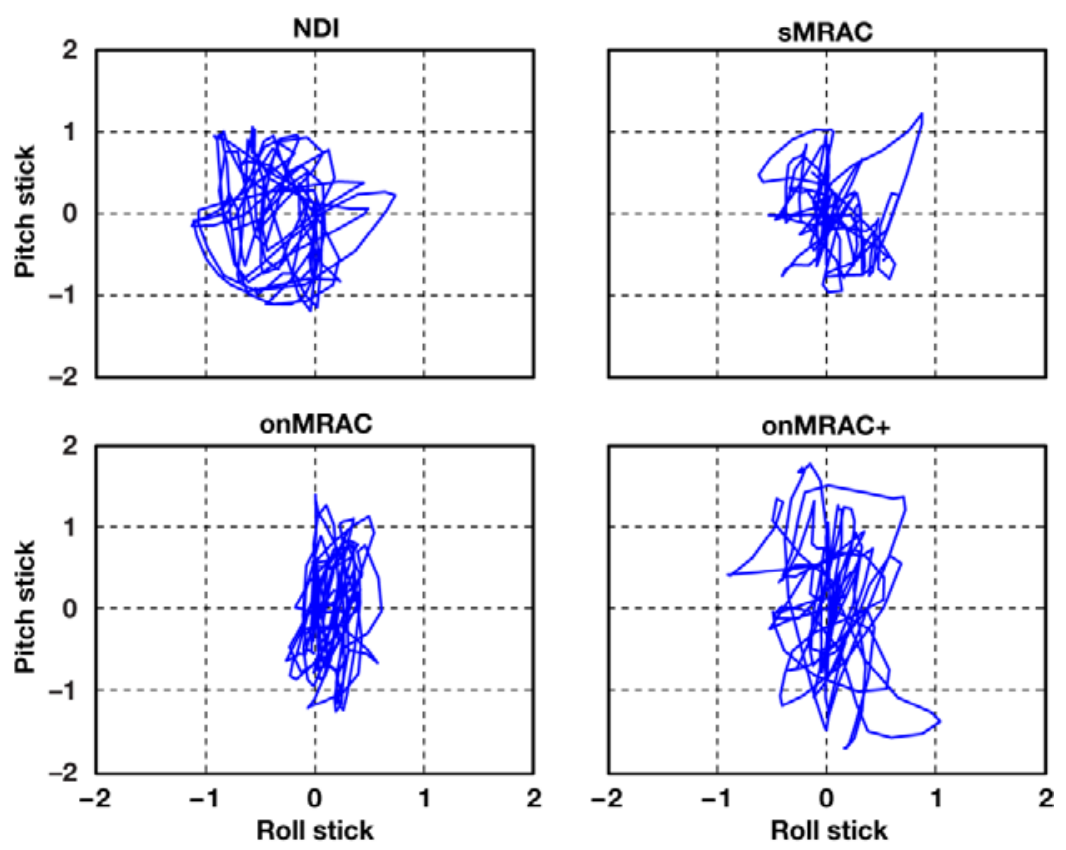

Figure 19. Input trace for Pilot $C$ during fine tracking task.

The tracking error metric for the pitch axis is shown in Fig. 20. The tracking error in pitch for the sMRAC is similar to the NDI; however, the tracking error in roll is reduced. This reduced roll tracking error likely explains the 
one $\mathrm{CH}$ rating improvement seen for this configuration. The onMRAC configuration is showing a rapid increase in error starting near $2.5 \mathrm{~s}$ into the task, which matches the stick trace showing something is occurring in the pitch axis.

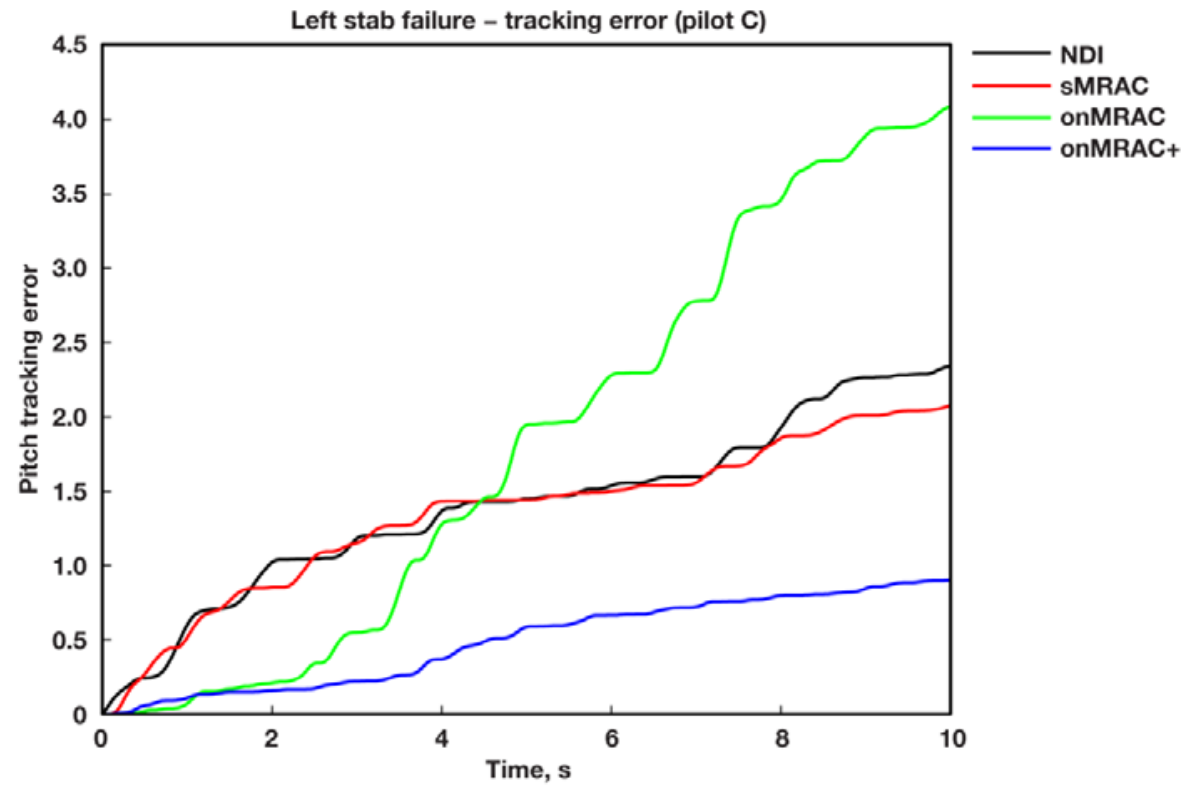

Figure 20. Pitch tracking error for Pilot C.

The reason for this increase in error is the result of the pitch adaptive parameter $\left(\hat{\theta}_{q 2}\right)$ growing fairly rapidly as shown in Fig. 21. During the gross acquisition task, the adaptive parameter went slightly negative. During the fine tracking task, $\hat{\theta}_{q 2}$ returns towards the value the sMRAC converged on; however, it oscillates by more than a value of one as seen in Fig. 21. These sharp changes in direction are a result of the normalization term. The onMRAC+ eliminated this effect for both pilots, the pitch adaptive term stayed negative, and the tracking error was seen to stay low and not grow as is seen in the onMRAC case.

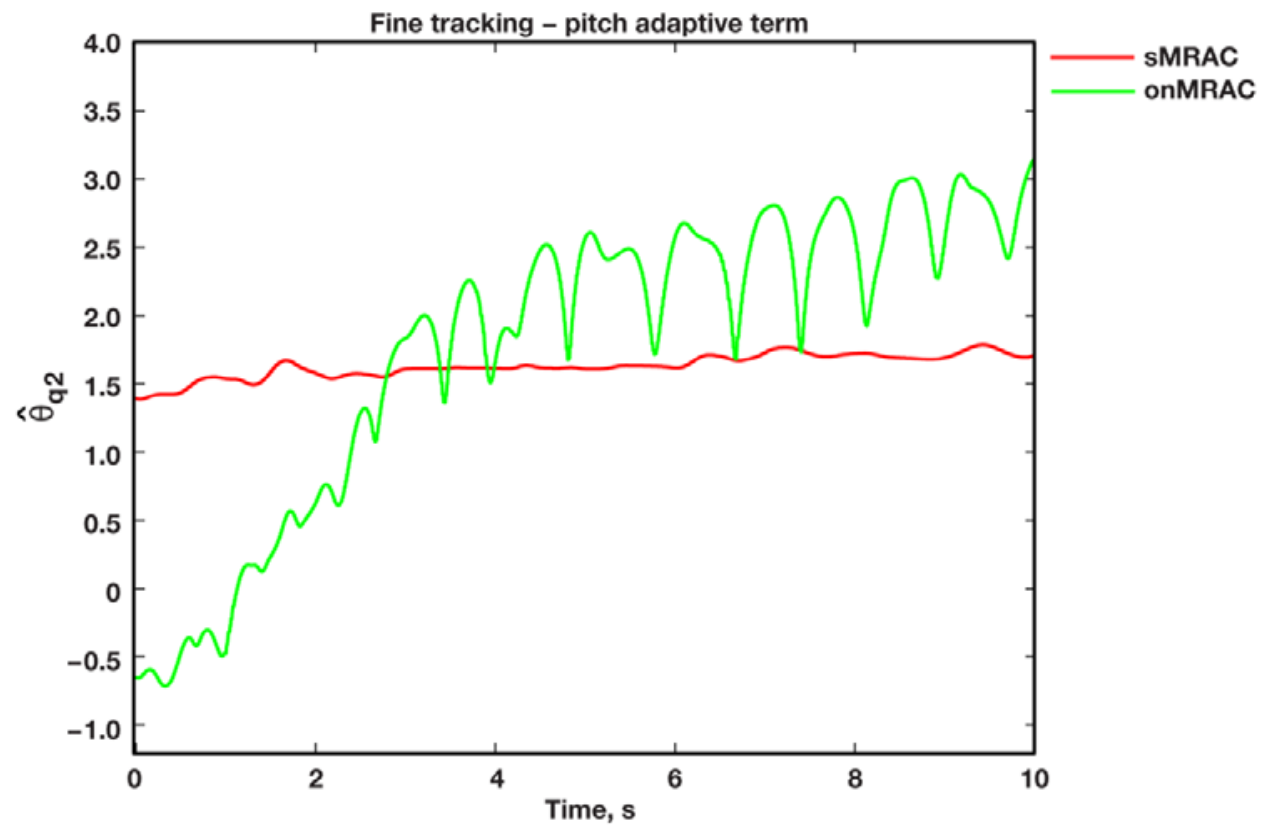

Figure 21. Matched pitch adaptive term during fine tracking task for Pilot C. 
The reason for the adaptive parameter behaving in this manner is a combined effect of the OCM term allowing for faster adaptation, and the normalization causing spiky behavior when the pilot's input is near or crosses zero. As the normalization is reduced, the learning rate is increased causing this behavior. This behavior resulted in a degradation of one $\mathrm{CH}$ rating from that of the sMRAC. The pilot commented that the reason for his rating of 4 was due to pilot compensation in pitch. Desired performance was still obtainable; however, the workload went from minimal to moderate. This increase in workload shows that the time varying nature of the pitch matched adaptive term is having a negative effect on the pilot. The onMRAC + did not show this same trend in tracking error as the onMRAC. The pitch adaptive parameter in this case was more level and never showed the time changing trend as seen with onMRAC. The extra adaptive term $\hat{\sigma}$ is reducing the error from this failure enough not to drive the matched adaptive term to grow as was seen for onMRAC case. The onMRAC+ did get the same ratings by this pilot as that of the onMRAC; however, the tracking error in both axes shows large improvement. This is a case where improved tracking error did not correlate to improved handling qualities. Simulation tests have shown that by changing the method of normalization and further tuning, the issues seen with the adaptive parameter can be greatly reduced.

\section{Complexity versus Performance Discussion}

The results given previously in this paper for the roll to pitch input coupling failure shows a clear trend between the complexity of the controller and the performance gain during a failure. As the complexity was increased in the controller, the amount of coupling was reduced and the handling qualities ratings generally got better. This improvement occurred to the point where they were equivalent to the baseline controller without a failure for the fine tracking task. For the left stab failure the results were mixed in relation to this tradeoff between complexity and performance. The handling qualities ratings showed no clear trend, but the tracking error showed improvement. The simplest MRAC was able to reduce the tracking error in roll while retaining the same error in pitch as the baseline controller. One pilot gave it better $\mathrm{CH}$ and PIO ratings than the NDI with the failure. For the onMRAC, there was an issue seen for one of the pilots where the adaptive parameter never settled on a single value. This issue posed a new problem of reducing the predictability of the controller. The last step in complexity added the time varying disturbance adaptive terms, which are more suited to handling coupled failures. This adaptive parameter was able to greatly reduce the tracking error for both failures.

Figure 22 shows an example plot of the overall tradeoff between performance and complexity for the three MRACs with the two failures. Here the performance metric is based on $\mathrm{CH}$ ratings averaged over all the pilots and both failures for the fine tracking task to give an overall assessment of each controller from the perspective of the pilots. Other metrics can be used, such as involving tracking error or other measures of performance. One issue with using tracking error as a sole metric is that it does not always correlate to handling qualities. This relation was seen in the roll to pitch input coupling failure where sMRAC and onMRAC were able to reduce coupling and improve handling qualities while having similar or even worse tracking error to that of the NDI with the failure. The same effect was also seen in the stab failure where onMRAC + had reduced tracking error in both axes, yet it had similar ratings to the NDI with the failure. 


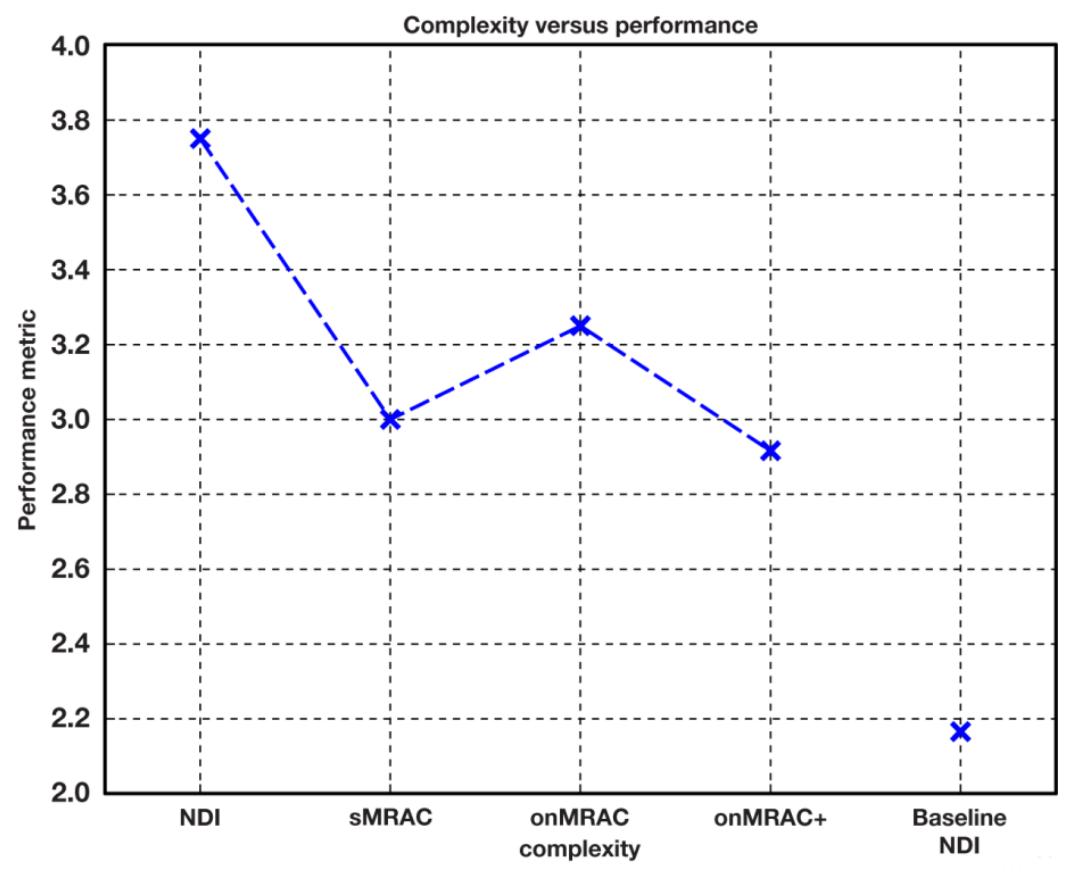

Figure 22. Complexity versus performance tradeoff.

For the x-axis in Fig. 22, the complexity is not well quantified. The approach in this paper was to incrementally add complexity, yet each step is not an equal jump in magnitude of complexity. Further research is needed to develop a metric to quantify complexity, the resulting development time, and $\mathrm{V} \& \mathrm{~V}$ effort required.

The data presented in Fig. 22 shows that a gain is achieved by the first step in complexity, sMRAC. This controller also likely represents the largest step in complexity as it initially adds the MRAC onto the NDI, which will be a large jump in the $\mathrm{V} \& \mathrm{~V}$ testing requirements. The onMRAC adds only changes to the update laws and shows slight degradation in overall performance driven by the issue seen with the left stab failed and the normalization term; however, this issue can be fixed as noted previously. With those fixes in place, the onMRAC+ would likely show even further improvement. By reducing the complexity of adaptive controllers, it may be easier to complete testing for certification requirements. The adaptive controllers tested here were able to show roughly a $50 \%$ performance improvement while remaining fairly simple with effectively just two to four adaptive parameters on only two feedback parameters (pitch rate and roll rate).

\section{Summary}

The approach taken in this paper was to use simple adaptive controllers and evaluate the performance as complexity was increased. Through flight tests on an F/A-18 aircraft with the left stabilator frozen and a roll to pitch input coupling failure, it was shown that the MRAC improved the performance of the aircraft, and the general trend was shown that with increased complexity comes increased performance. Complexity, however, can add to the required effort to $\mathrm{V} \& \mathrm{~V}$ test for certification so a tradeoff must be made. The onMRAC+ configuration adapted sufficiently and brought the controller back towards the baseline controller, while still being fairly simple. Even the sMRAC was able to achieve an improvement in handling qualities for the failure conditions, however, it had a limited ability to remove coupling. The controllers tested in this paper were simple yet effective in reducing the effects of failures. 


\section{References}

${ }^{1}$ Pavlock, K., "Full-Scale Advanced Systems Testbed: Ensuring Success of Adaptive Control Research Through Project Lifecycle Risk Mitigation,” SFTE 2011 (to be published), 2011.

${ }^{2}$ Krishnakumar, K., "A Request for Information in Flight Validation of Adaptive Control to Prevent Loss-of-Control Events," Solicitation Number: NNH09ZEA002L, NASA Headquarters Office of Procurement, April 2009.

${ }^{3}$ Jacklin, S., "Closing the Certification Gaps in Adaptive Flight Control Software," AIAA-2008-6988, 2008.

${ }^{4}$ Miller, C. J., "Nonlinear Dynamic Inversion Baseline Control Law: Architecture and Performance Predictions," AIAA-2011-\#\#\# (to be published), 2011.

${ }^{5}$ Ioannou, P. A., and Sun, J., Robust Adaptive Control, Prentice Hall, Inc., Upper Saddle River, New Jersey, 1995.

${ }^{6}$ Hanson, C., Johnson, M., and Schaefer, J., "Design of a Low Complexity Model Reference Adaptive Controller," NASA/TM-\#\#\#\#-215972 (to be published).

${ }^{7}$ Nguyen, N., Krishnakumar, K., and Boskovic, J., “An Optimal Control Modification to Model-Reference Adaptive Control for Fast Adaptation," AIAA-2009-7283, 2009.

${ }^{8}$ Hanson, C., Schaefer, J., Johnson, M., and Nguyen, N., "Handling Qualities Evaluations of Low Complexity Model Reference Adaptive Controllers for Reduced Pitch and Roll Damping Scenarios” AIAA-2011-\#\#\#\# (to be published), 2011.

${ }^{9}$ Fields, D., Marten, D., Di Loreto, G., Koo, R., Lemery, J., and Ryan, K., "Limited Handling Qualities Evaluation of Inter-axis Control Coupling (Project Icarus)," AFFTC-TIM-10-03, June 2010.

${ }^{10}$ Cooper, G. E., and Harper, R. P., "The Use of Pilot Rating in the Evaluation of Aircraft Handling Qualities," NASA/TN D-5153, April 1969.

${ }^{11}$ U. S. Department of Defense, "Flying Qualities of Piloted Aircraft,” MIL-STD-1797B, 15 February 2006.

${ }^{12}$ Gray, W. R., "Handling Qualities Evaluation at the USAF Test Pilot School," AIAA-2009-6317, 2009.

${ }^{13}$ United States Army Aviation and Missile Command, Aeronautical Design Standard, "Handling Qualities Requirements for Military Rotorcraft," ADS-33E-PRF, 21 March 2000. 


\section{Handling Qualities of Model Reference Adaptive Controllers with Varying Complexity for Pitch-Roll \\ Coupled Failures}

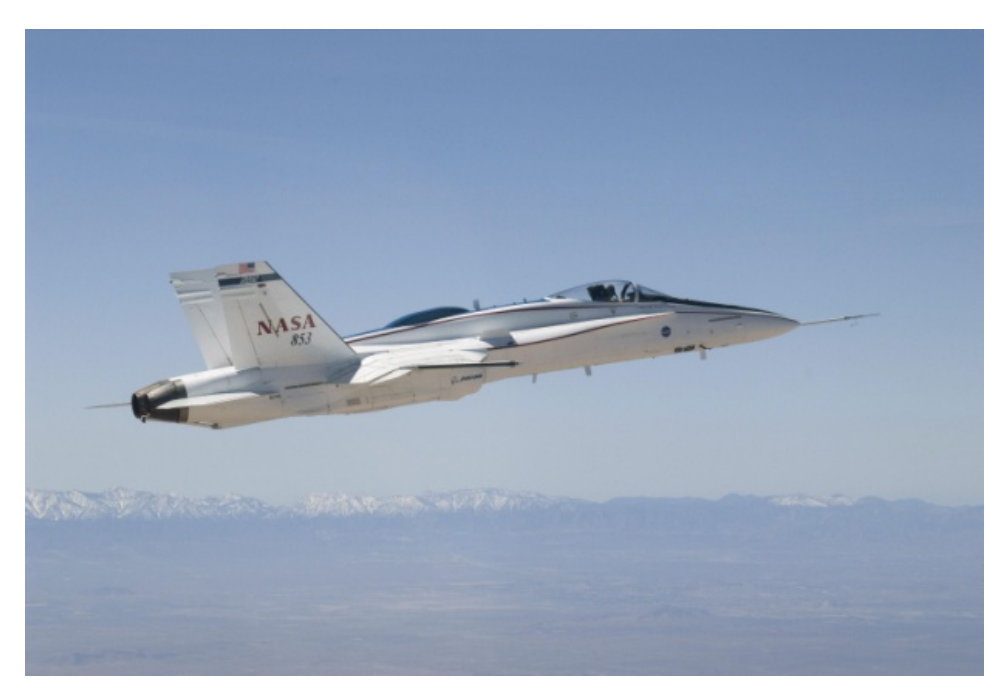

Jacob Schaefer 


\section{Outline}

- Background

- Description of the Model Reference Adaptive Controller

- Failure Scenarios

- Handling Qualities Tasks

- Results

- Summary 


\section{Background}

- In 2009, NASA released a Request For Information (RFI)

- "In Flight Validation of Adaptive Control to Prevent Loss of Control Events"

- Results from Government, Industry, and Academia highlighted three areas of focus

1. Investigate simple yet effective adaptive control

2. Explore pilot interaction with adaptive control

3. Incorporate structural feedback into fight control system

- Research presented here focuses primarily on \#1 


\section{Background}

- Objective was to perform flight research on simple adaptive controllers to investigate the relationship between complexity and performance.

- Complexity here means the number of feedback loops, nonlinear elements, adaptive gains, tunable gains, etc.

- Simplification from previous work at NASA on the Intelligent Flight Control System (IFCS)

- Flight research performed on NASA's Full-scale Advanced Systems Testbed (an F/A-18) which has a research flight control system. 


\section{Background}

\section{Example of simplification from IFCS to current MRAC}

\section{IFCS Pitch Axis Adaptive Terms}

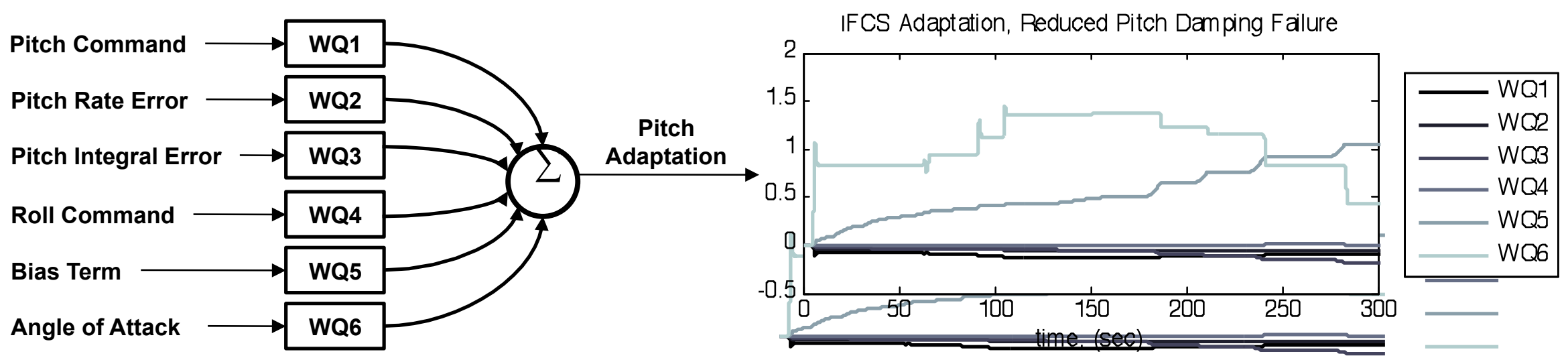

Current MRAC Pitch Axis Adaptive Terms onMHAC+ Adaptatlon, Heduced Hitch vamping ralure

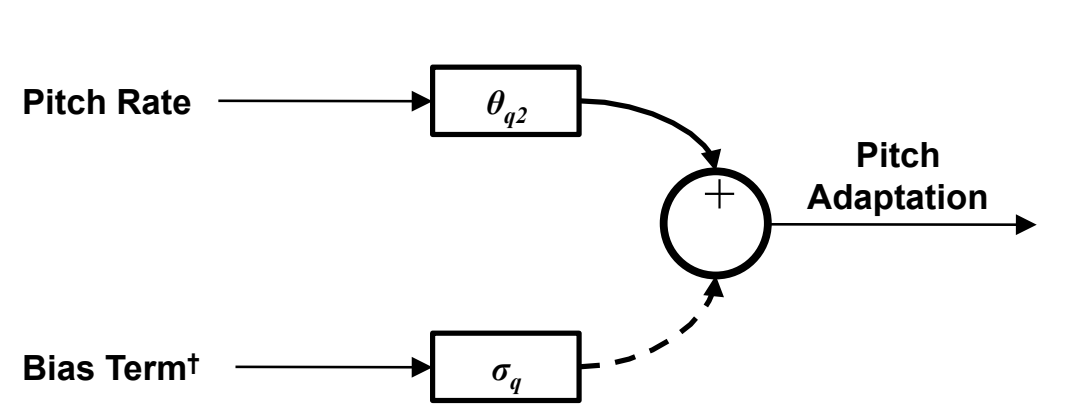

tThe Bias Term was only active in one of the three MRACs tested

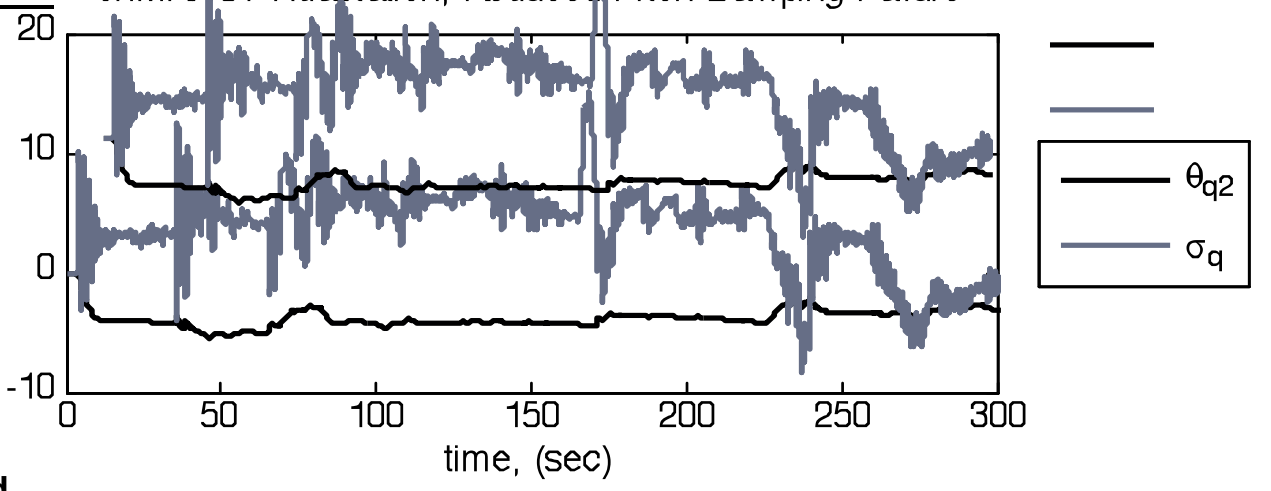




\section{Design}

- Baseline Controller - Nonlinear dynamic inversion (NDI) controller

- Gives aircraft dynamics that match reference model under nominal flight

- Reference models chosen to give good handling qualities

- Model Reference Adaptive Controller (MRAC)

- Additive dynamics into dynamic inversion in pitch and roll axes only

Nonlinear Dynamic Inversion Controller

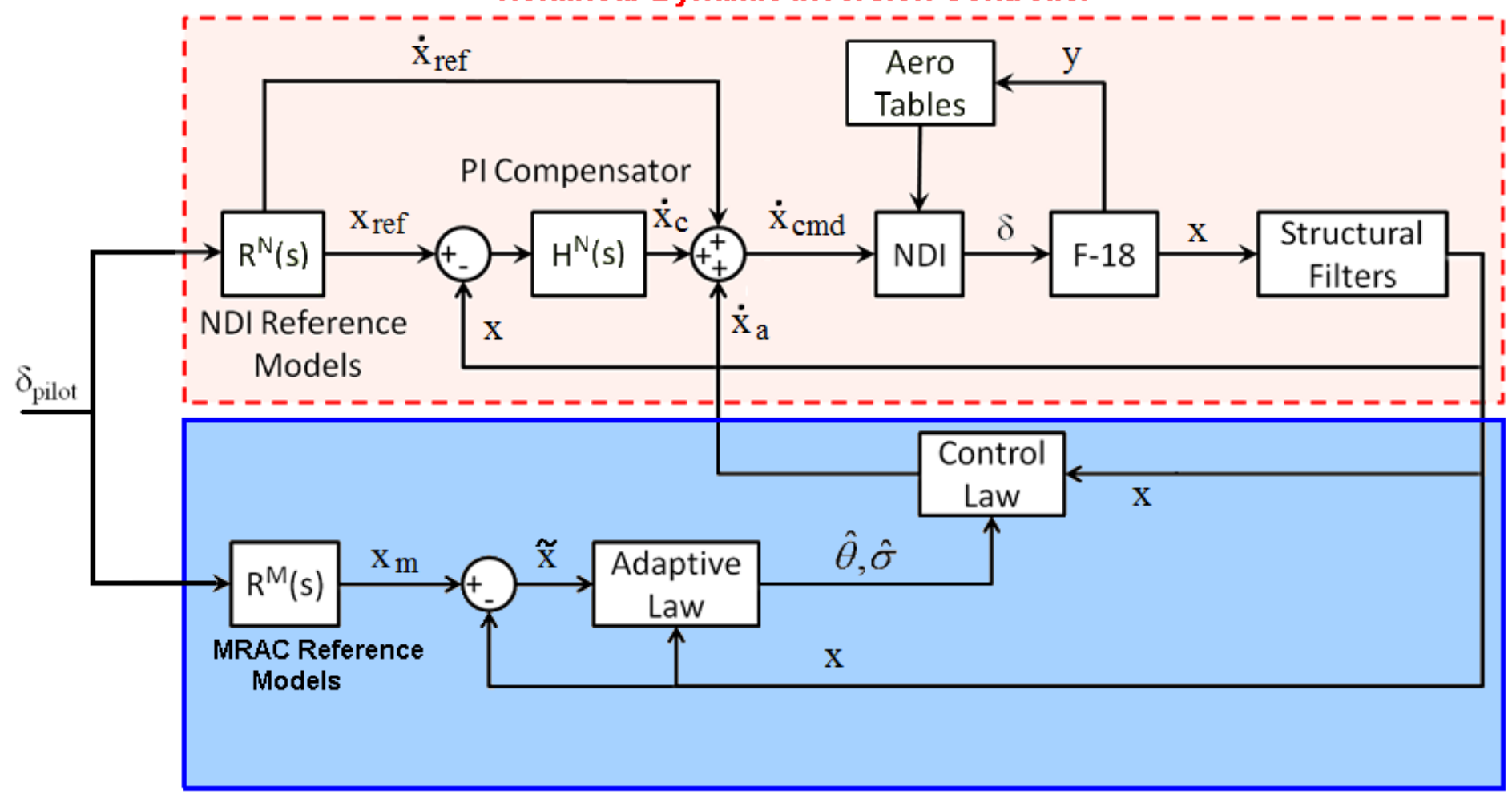

Model Reference Adaptive Controller 


\section{MRAC Design}

\section{Reference Models}

$\frac{\dot{p}_{r e f}}{r_{p}}=\frac{s}{s+\omega_{p}} \quad \frac{\dot{q}_{r e f}}{r_{q}}=\frac{s^{2}}{s^{2}+2 \zeta_{q} \omega_{q} s+\omega_{q}^{2}}$

$$
\begin{gathered}
\text { MRAC Error } \\
\tilde{x}_{p}(t)=p_{m}(t)-p(t) \\
\tilde{x}_{q}(t)=\left[\begin{array}{c}
\int q_{m}(t)-\int q(t) \\
q_{m}(t)-q(t)
\end{array}\right]
\end{gathered}
$$

Aircraft dynamics with uncertainty and adaptive terms

$$
\begin{gathered}
\dot{x}(t)=A_{m} x(t)+B_{m} r(t)+B_{m}\left(\widehat{\Theta}^{\mathrm{T}}(t)-\Theta^{\mathrm{T}}\right) x(t)+B_{m}(\hat{\sigma}(t)-\sigma(t)) \\
\text { as } \widehat{\Theta}(t) \rightarrow \Theta, \hat{\sigma}(t) \rightarrow \sigma \\
\dot{x}(t)=A_{m} x(t)+B_{m} r(t)
\end{gathered}
$$

MRAC Control Law

$$
\dot{x}_{a_{p}}=\hat{\theta}_{p} p+\hat{\sigma}_{p} \quad \dot{x}_{a_{q}}=\hat{\theta}_{q 1} \int q d t+\hat{\theta}_{q 2} q+\hat{\sigma}_{q}
$$




\section{MRAC Design}

\section{Three MRACs of varying complexity}

- sMRAC : Simplest MRAC (2 matched adaptive terms)

$$
\dot{\hat{\theta}}_{p}=\Gamma_{\mathrm{p}} \tilde{x}_{p} P_{p} B_{m_{p}} p \quad\left[\begin{array}{c}
\hat{\hat{\theta}}_{q 1} \\
\hat{\hat{\theta}}_{q 2}
\end{array}\right]=\left[\begin{array}{cc}
\Gamma_{\mathrm{q} 1} & 0 \\
0 & \Gamma_{\mathrm{q} 2}
\end{array}\right] \tilde{x}_{q}^{T} P_{q} B_{m_{q}} x_{q}
$$

Note: effect of $\int q$ was eliminated, making $\hat{\theta}_{q 1}$ negligible

- onMRAC : Optimal control modification and Normalization

$$
\begin{gathered}
\dot{\hat{\theta}}_{p}=\frac{\Gamma_{\mathrm{p}}}{1+R_{\theta_{p}} p^{2}}\left(\tilde{x}_{p} P_{p} B_{m_{p}} p-v_{\theta_{p}} p^{2} \hat{\theta}_{p} B_{m_{p}}^{T} P_{p} A_{m_{p}}^{-1} B_{m_{p}}\right) \\
{\left[\begin{array}{c}
\hat{\theta}_{q 1} \\
\hat{\theta}_{q 2}
\end{array}\right]=\frac{\left[\begin{array}{cc}
\Gamma_{\mathrm{q} 1} & 0 \\
0 & \Gamma_{\mathrm{q} 2}
\end{array}\right]}{1+x_{q}^{T} R_{\theta_{q}} x_{q}}\left(\tilde{x}_{q}^{T} P_{q} B_{m_{q}} x_{q}-x_{q} v_{\theta_{q}} T_{q}^{T}\left[\begin{array}{l}
\hat{\theta}_{q_{1}} \\
\hat{\theta}_{q_{2}}
\end{array}\right]_{m_{q}}^{T} P_{q} A_{m_{q}}^{-1} B_{m_{q}}\right)}
\end{gathered}
$$

- onMRAC+ : Additional adaptive terms

$$
\begin{gathered}
\dot{\hat{\sigma}}_{p}=\frac{\Gamma_{\sigma_{p}}}{1+R_{\sigma_{p}} p^{2}}\left(\tilde{x}_{p} P_{p} B_{m_{p}}-v_{\theta_{p}} \hat{\sigma}_{p} B_{m_{p}}^{T} P_{p} A_{m_{p}}^{-1} B_{m_{p}}\right) \\
\dot{\hat{\sigma}}_{q}=\frac{\Gamma_{\sigma_{\mathrm{q}}}}{1+x_{q}^{T} R_{\sigma_{q}} x_{q}} \tilde{x}_{q}^{T} P_{q} B_{m_{q}}
\end{gathered}
$$




\section{Failure Scenarios}

- Roll to Pitch input coupling (RxP)

- Adds only pitch due to roll

- Pitch Input = Pitch Input + Roll Input

- Left Stabilator Failure

- Freezes left stab at 0 degrees deflection

- Off trim by a couple degrees at flight condition

- Causes cross coupling between axes 


\section{Flight Research}

- Maneuvers - Doublets, captures, steady heading sideslips, 360 degree rolls, $2.5 \mathrm{~g}$ windup turns, $2 \mathrm{~g}$ loaded roll reversals

- Random ordering of controller configurations tested

- Handling Qualities Task (2 Tasks)

- In trail formation flight

- Gross Acquisition of wingtip, then fine tracking of wingtip

Gross acquisition / fine tracking maneuver illustration

\section{Test aircraft canopy}

mark aligned with

lead aircraft tail hook 


\section{RxP Results}

- Roll to pitch input coupling

- Response to pitch doublet then roll doublet

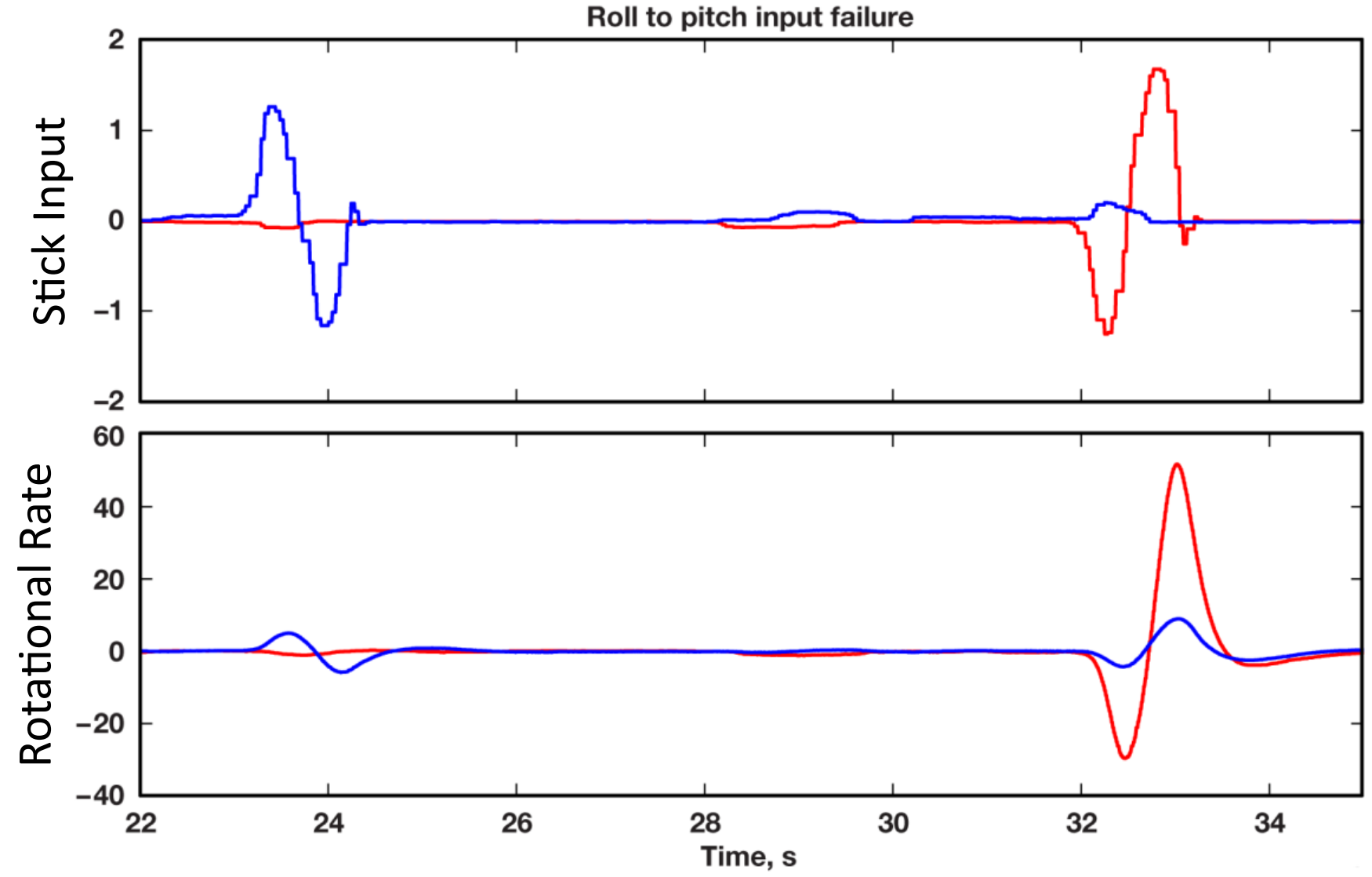




\section{Results - RxP}

Roll to Pitch Input Coupling - Cooper-Harper and PIO ratings
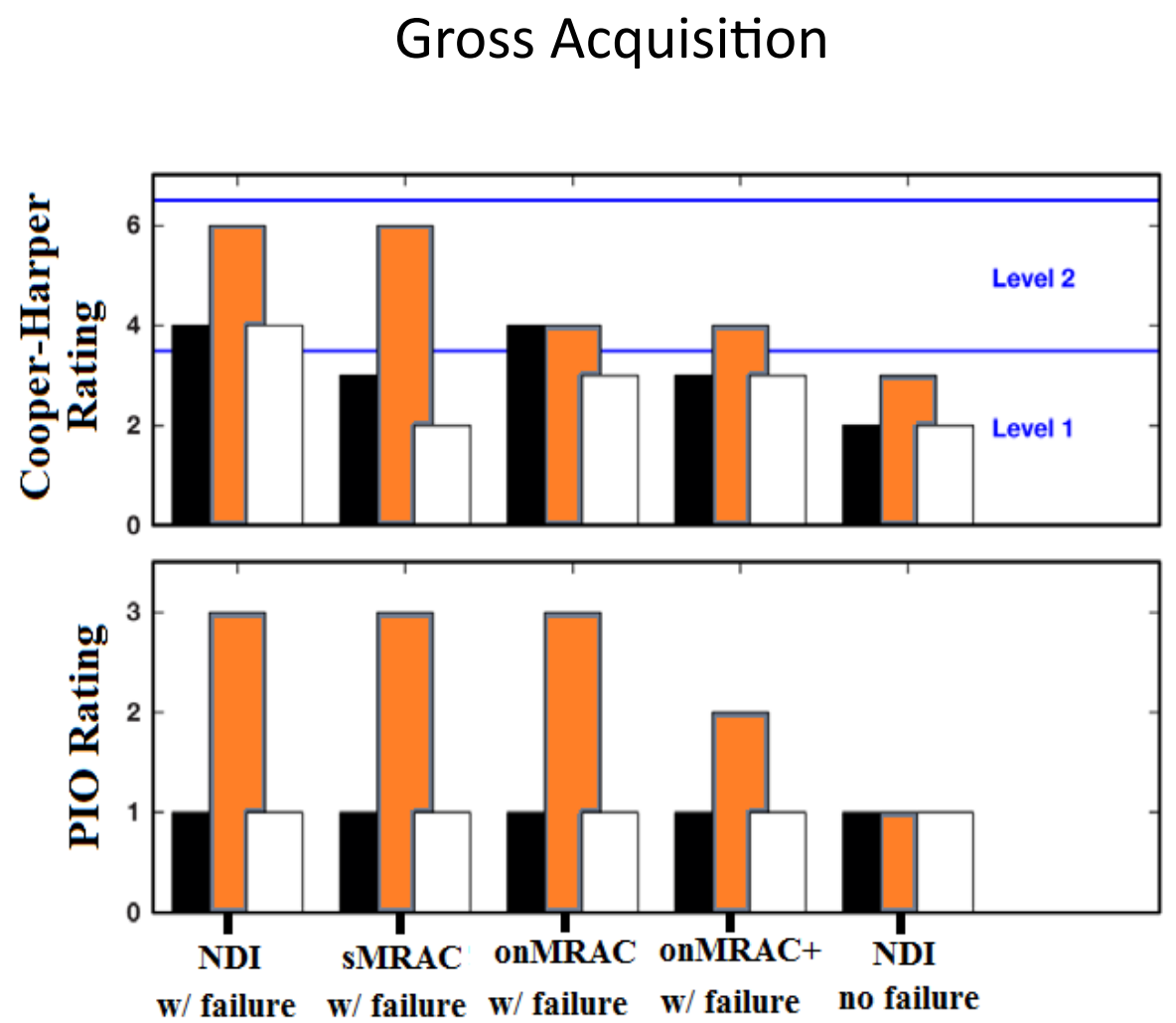

Fine Tracking

Pilot A
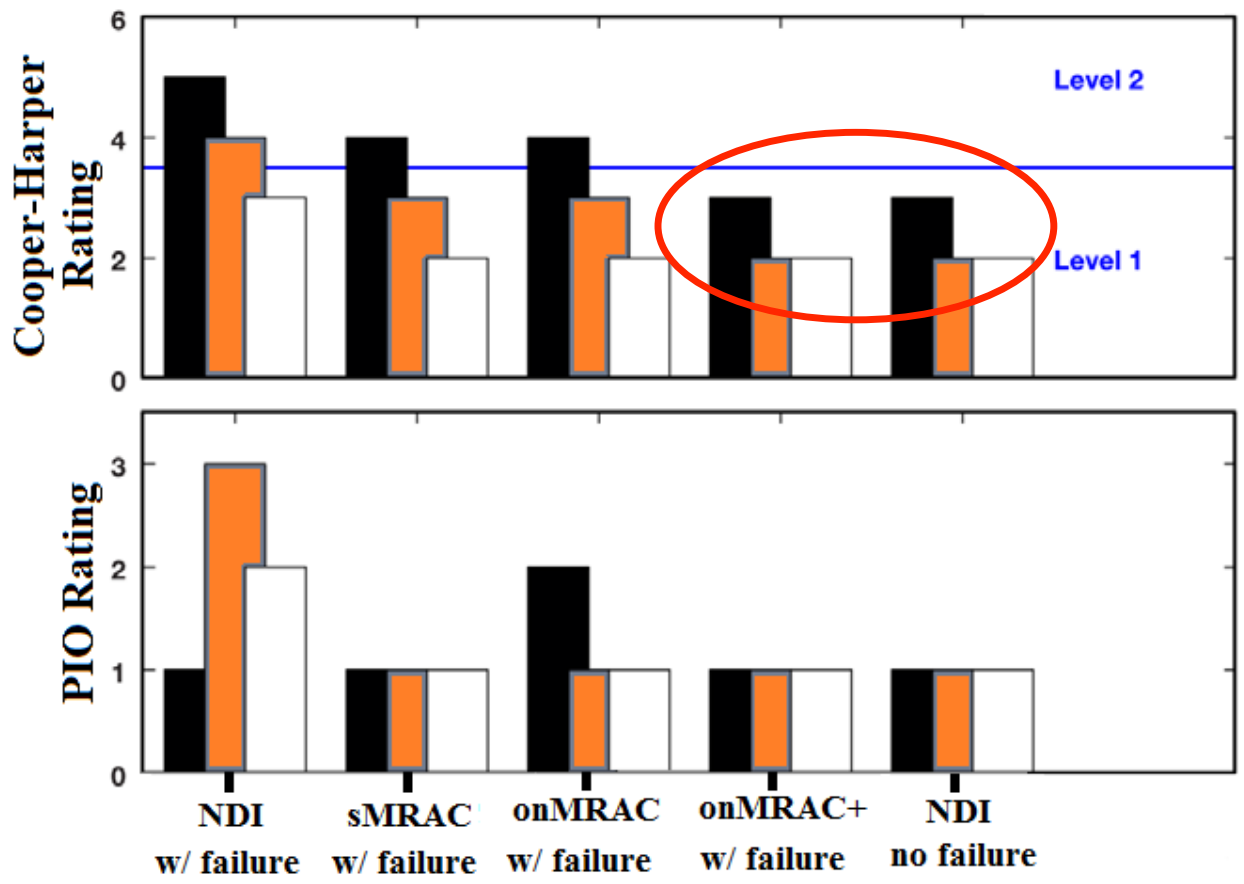


\section{Results - RxP}

- Magnitude of coupling of q/ $p$ with $\mathrm{HQ}$ ratings from fine tracking task

- ADS-33E line is for helicopter cross coupling

- Icarus line is from a recent study on an F-16 using cross coupling

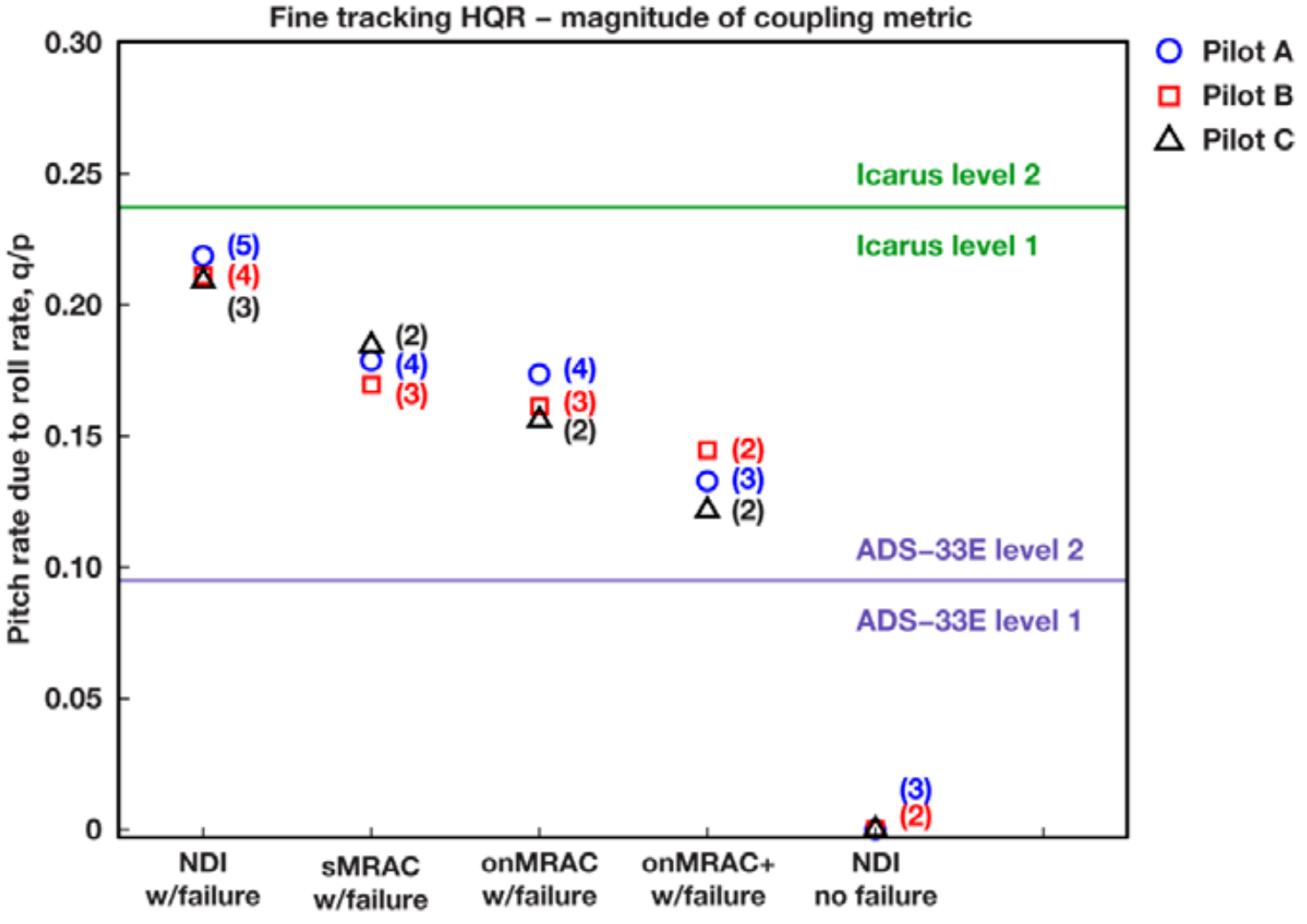




\section{Results - RxP}

- Traces of stick input for Pilot A during 15 seconds of fine tracking task

- Red line is linear fit to average longitudinal input per lateral input

- Pilot must compensate for coupling that was not removed by the adaptive controller
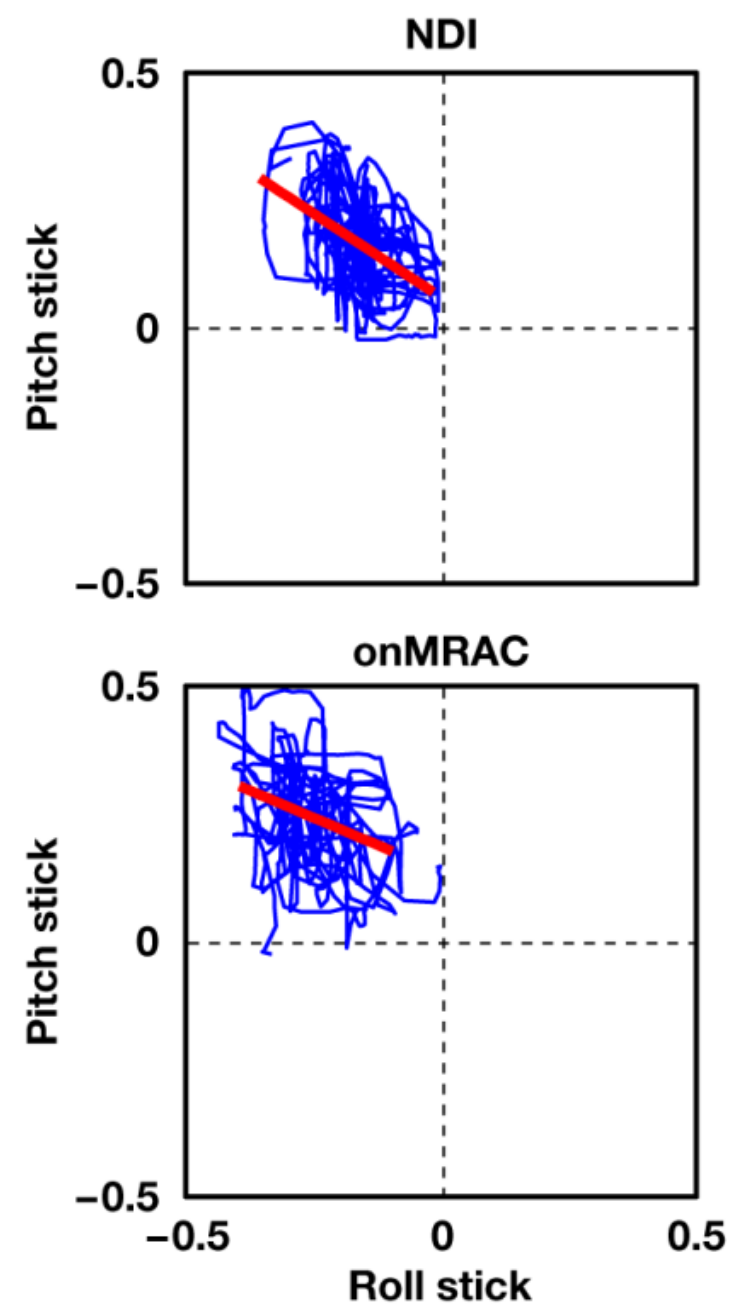

SMRAC

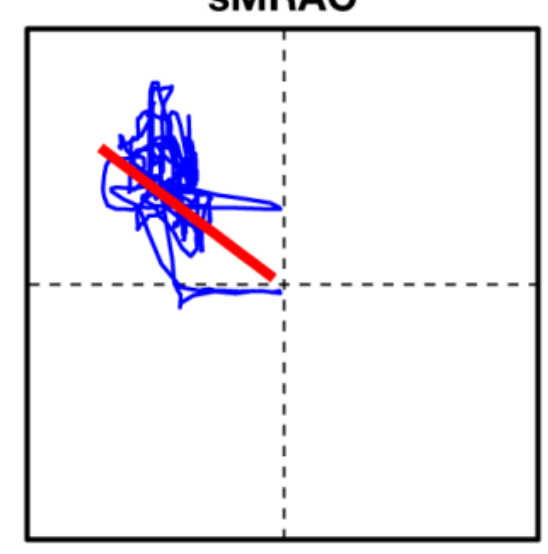

OnMRAC+

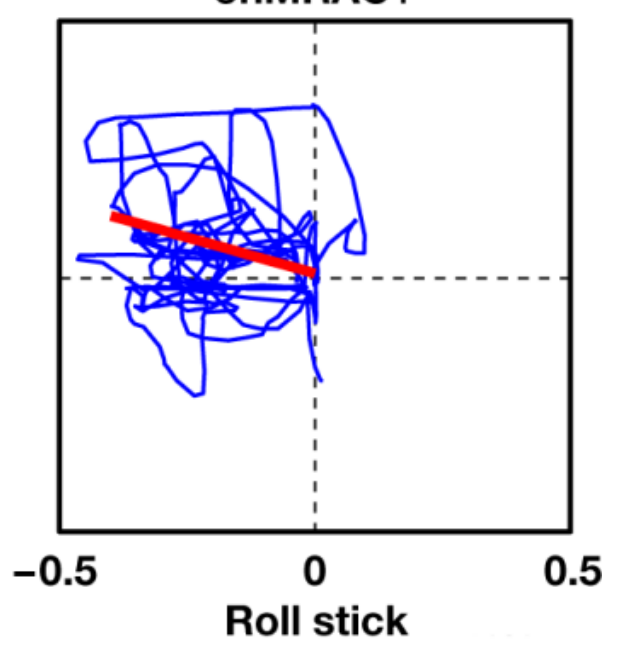




\section{Results - Stab}

- Left Stabilator frozen at zero degrees deflection

- Response to Pitch doublet then roll doublet
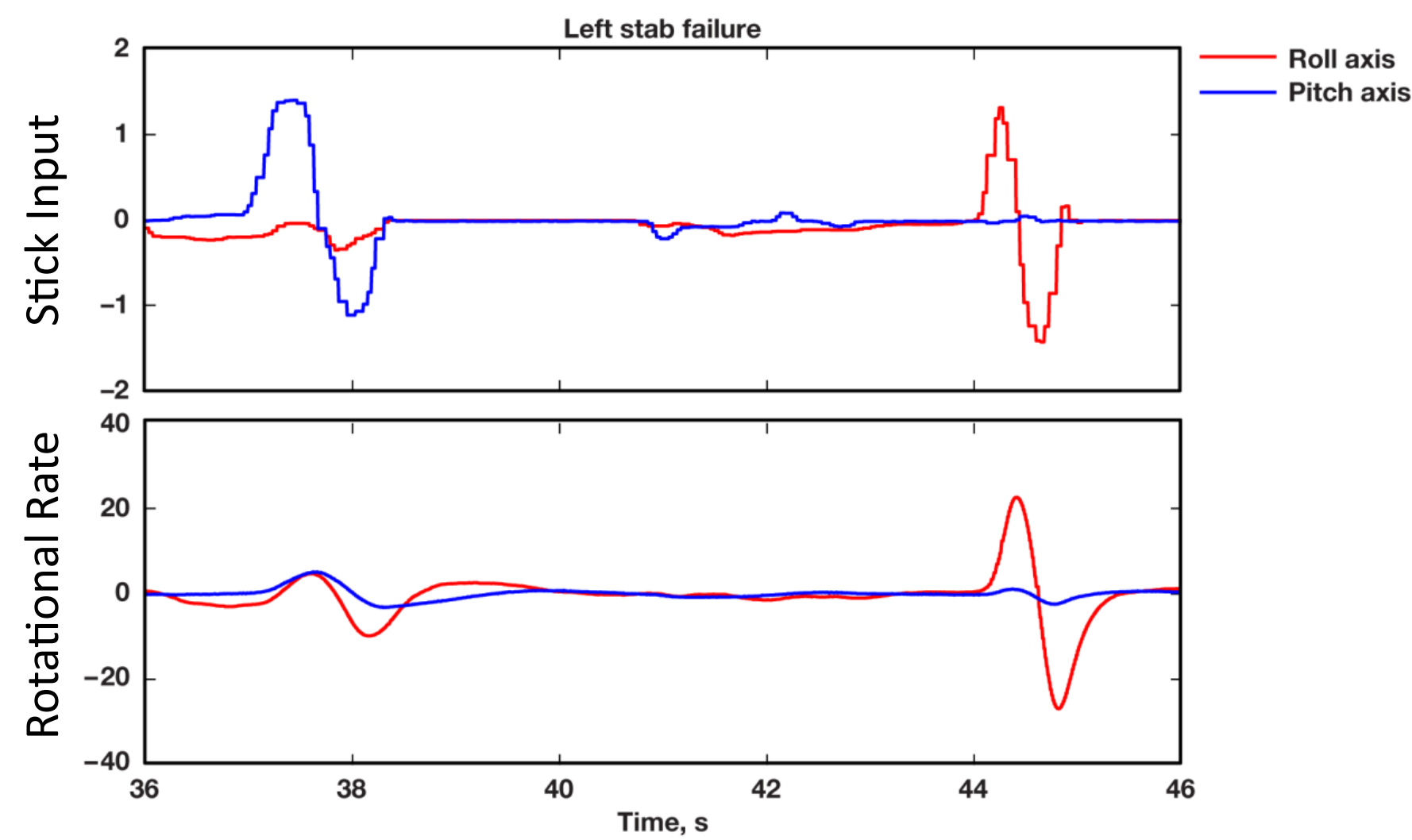


\section{Results - Stab}

- Handling qualities ratings show little change

- Pilot D's comments however got better. NDI with failure was sluggish while onMRAC+ felt more sensitive and like a "normal Hornet"

- Pilot C felt the plane was sluggish for all controllers with the failure

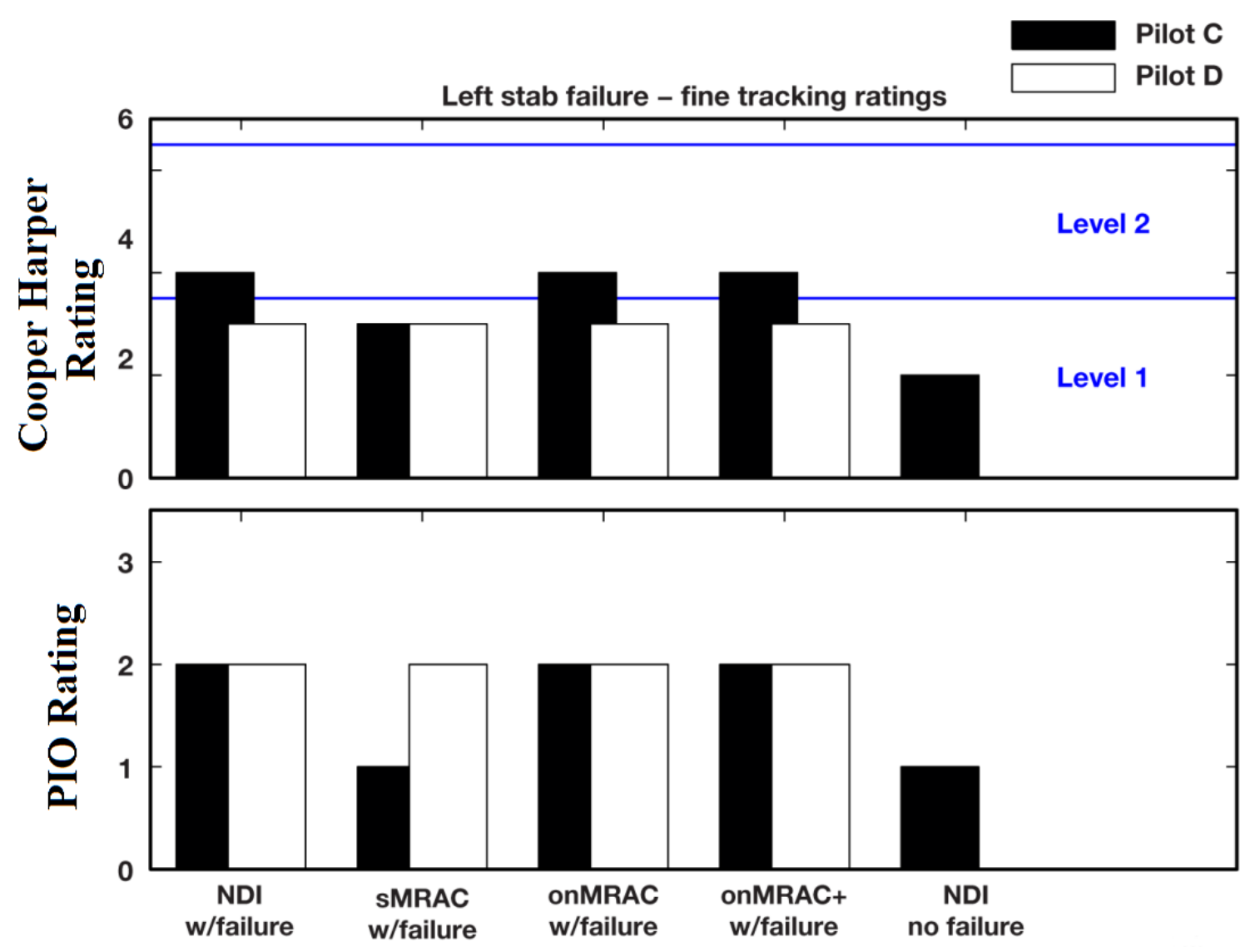




\section{Results - Stab}

- MRAC tracking error for Pilot $C$

- sMRAC, roll improvement

- onMRAC, pitch issue

- onMRAC+, large improvement yet no rating improvement

\section{Pitch}

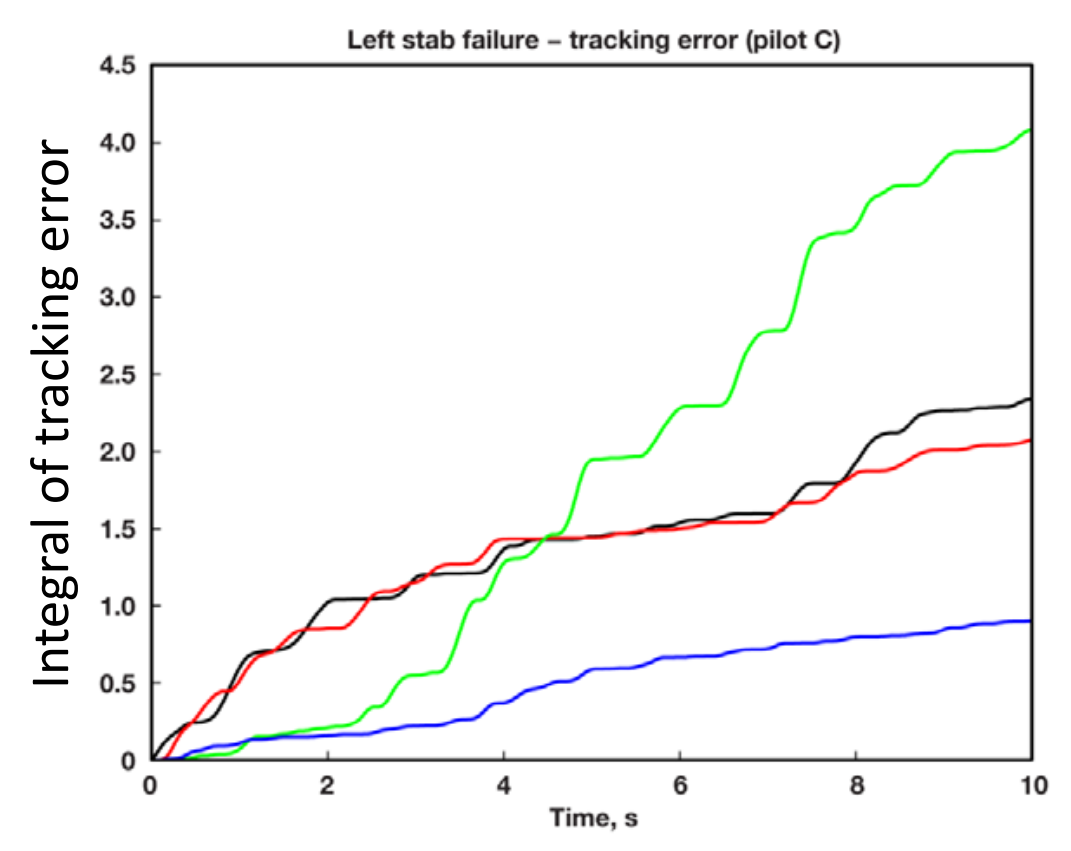

$\underline{\text { Roll }}$

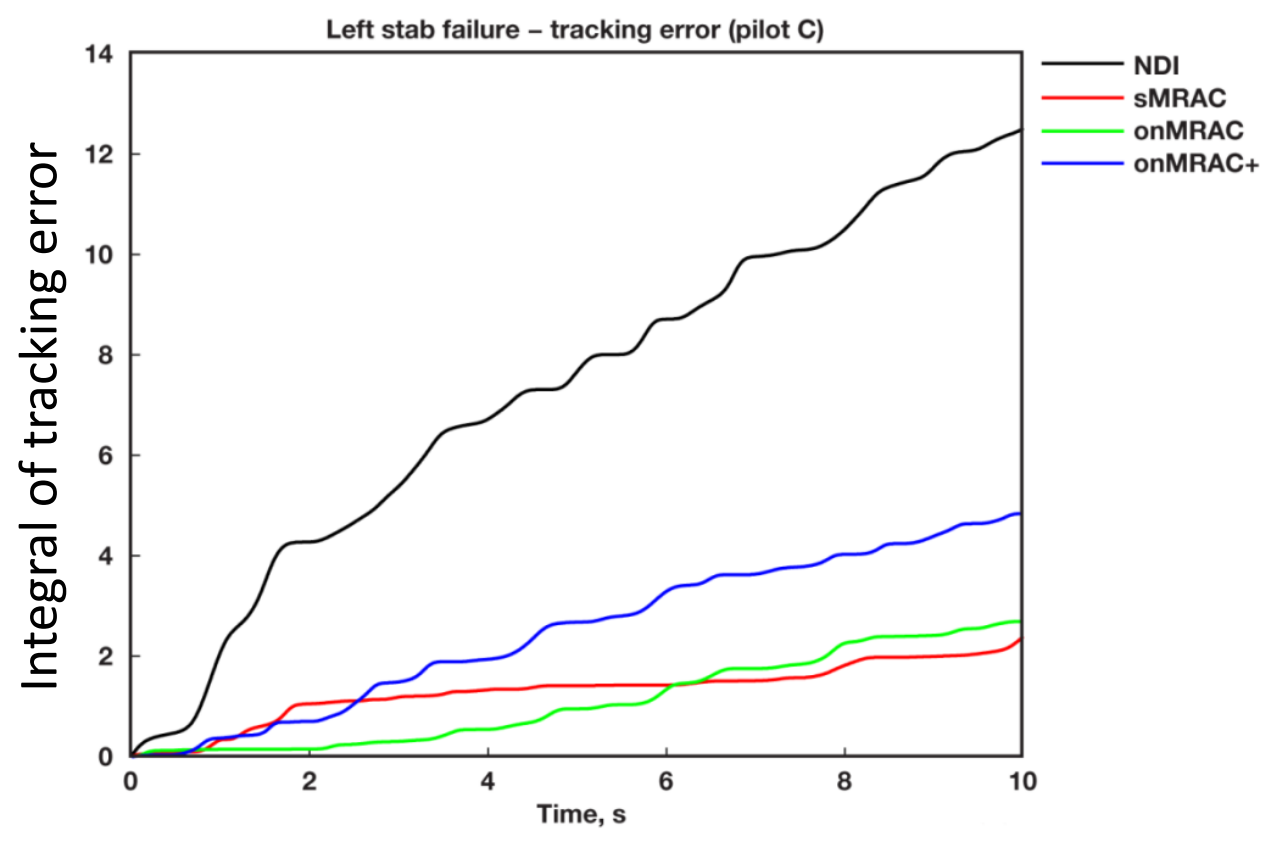




\section{Results - Stab}

- Pilot commented on pitch having poor predictability and that it felt "weird" in pitch (very high gain and aggressive pilot)

- Unmatched failure, but the pitch adaptive term should go positive to add pitch effectiveness to compensate for loss of one stab

- Very aggressive pilot maneuvers generating large error and the normalization did not have a large enough effect of reducing learning rate which allowed for this spiky behavior.

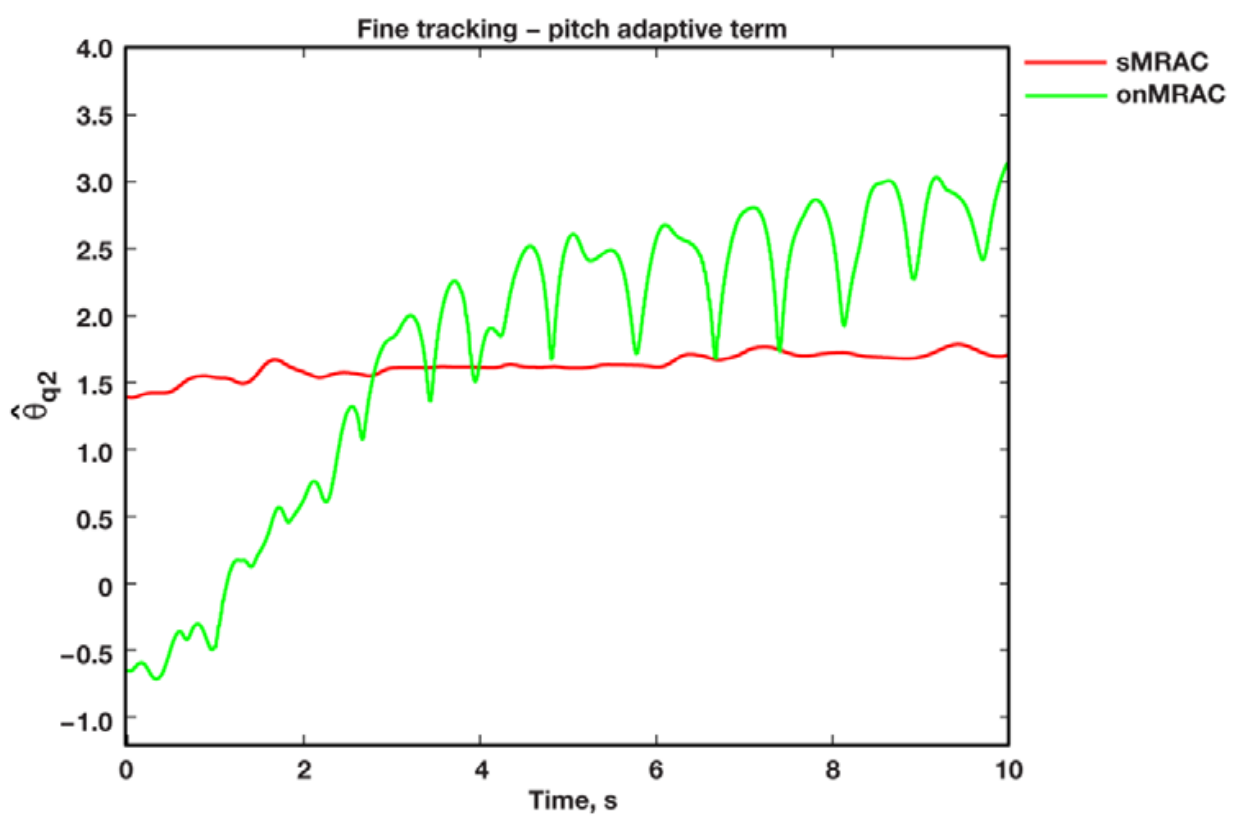

- Simulation studies show this can be fixed

by increasing normalization term

$$
\dot{\hat{\theta}}_{q 2}=\frac{\Gamma_{\mathrm{q} 2}}{1+R_{\theta_{q}} q^{2}}\left(\tilde{x}_{q} P_{q} B_{m_{q}} q-v_{\theta_{q}} q^{2} \widehat{\theta}_{q_{2}} B_{m_{q}}^{T} P_{q} A_{m_{q}}^{-1} B_{m_{q}}\right)
$$




\section{Results Summary}

- Roll to pitch input coupling scenario showed steady improvement with added complexity

- $50 \%$ of coupling removed

- As complexity was added, the average Cooper Harper rating either remained the same or got better for all cases

- For the fine tracking task, onMRAC+ with the failure had the same ratings as the healthy aircraft with NDI

- Left stab failure showed little improvement in ratings however it was not a severe failure

- One pilot thought most complex controller felt more like normal aircraft and sluggishness was removed

- Other pilot had predictability issue with onMRAC, and thought things stayed generally the same for all controllers 


\section{Conclusions}

- The three MRACs were all able to either make things better or at the least stay the same as the NDI with the failure.

- A sacrifice in performance can be made by reducing complexity such as from the onMRAC+ which had 4 adaptive terms and was able to reduce coupling by $50 \%$ to that of the SMRAC which had 2 adaptive terms with basic update laws and could reduce $25 \%$ of the coupling.

- A control system designer can make this tradeoff to more easily and more realistically meet the constraints of the implementation and $V \& V$ requirements and at least get some level of performance improvement which will help the pilot in the presence of a failure and could potentially prevent a loss of control event which he/she would not have been able to avoid if there was no adaptive system. 
Questions? 